\title{
CONTEMPORARY ECONOMICS
}

Quarterly of University of Finance and Management in Warsaw

Volume 5 Issue 3

September 2011

SPECIAL ISSUE

\section{SOCIAL DIAGNOSIS 2011}

OBJECTIVE AND SUBJECTIVE QUALITY OF LIFE IN POLAND

\author{
REPORT
}

Edited by

Janusz Czapiński

Tomasz Panek

Warsaw: The Council for Social Monitoring 


\section{CONTEMPORARY ECONOMICS}

ABSTRACTED/INDEXED:

- Cabell's Directories

- ECONIS

- EconStor

- GALE Science in Context

- Ministry of Science and Higher Education list of scored journals (rating score 9 pts)

- Research Papers in Economics (RePEc)

- SCOPUS

- The Central European Journal of Social Sciences and Humanities

- The International Bibliography of the Social Sciences (IBSS)/ProQuest

- Ulrichsweb

Contemporary Economics is published with the financial support of Polish Ministry of Science and Higher Education, in the frame of research supporting activity and programme INDEX PLUS.

\section{Academic Board}

Icek Ajzen (USA)

Damodaran Appukuttan Nair (India)

Zenon Biniek (Poland)

Constantin A. Bob (Romania)

Wiesław Dębski (Poland)

Bruno S. Frey (Switzerland)

Masahiko Gemma (Japan)

Kjell Åge Gotvassli (Norway)

Adriana Grigorescu (Romania)

Zoran Ivanovic (Croatia)

Sten Jönsson (Sweden)

Victor Martinez Reyes (USA)

Ieva Meidute (Lithuania)

Fatmir Memaj (Albania)

Grażyna Rytelewska (Poland)

Shelby D. Hunt (USA)

Maria Sierpińska (Poland)

President of Academic Board

Miemie Struwig (South Africa)

Tadeusz Szumlicz (Poland)

\section{Editorial Board}

Witold Jakóbik

Henryk Król

Editor in Chief

Witold Małecki

Danuta Mliczewska

Deputy Editor in Chief

Włodzimierz Rembisz

Marcin Staniewski

Deputy Editorial Manager

Piotr Szczepankowski

Editorial Manager

Ryszard Wilczyński

\section{Address of Editors:}

\section{CONTEMPORARY ECONOMICS}

University of Finance and Management in Warsaw

01 - 030 Warsaw, 55 Pawia Str., room 211, phone: (22) 5365454

e-mail: editorial@ce.vizja.pl

www.ce.vizja.pl

Publisher:

Vizja Press \& IT

01 - 029 Warsaw, 60 Dzielna Str.

phone/fax: (22) 5365468

e-mail: vizja@vizja.pl

www.vizja.net.pl

All articles published in the quarterly are subject to reviews 
The opinions and conclusions contained herein represent the authors' personal views and do not necessarily reflect the official position of the Ministry of Labour and Social Policy.

The following publication was prepared as part of the Social Diagnosis 2009-2013 system project realised by the Human Resources Development Fund (CRZL) and initiated by the Department of Economic Analyses and Forecasts (Ministry of Labour and Social Policy).

Translation and typesetting:

Contact Language Services Sp. J.

Proofreading:

1. Contact Language Services Sp. J.

2. Emil Tchorek

SBN 978-83-61638-30-8

The following publication was co-financed by the European Union within the framework of the European Social Fund.

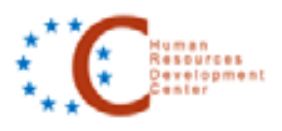




\title{
Research project designed by
}

THE COUNCIL FOR SOCIAL MONITORING:

Dominik Batorski, Ph. D., University of Warsaw

Professor Janusz Czapiński, Ph. D., University of Warsaw and University of Finance and Management (chairman)

Professor Janusz Grzelak, Ph. D., University of Warsaw

Teresa Kamińska, M. Sc., PRO PUBLICO BONO Institute

Professor Irena E. Kotowska, Ph. D., Warsaw School of Economics

Wiesław Łagodziński, M. Sc., Central Statistical Office (secretary)

Professor Tomasz Panek, Ph. D., Warsaw School of Economics (vice-chairman)

Professor Antoni Sułek, Ph. D., University of Warsaw

Professor Tadeusz Szumlicz, Ph. D., Warsaw School of Economics

\author{
Authors of the report: \\ Dominik Batorski \\ Piotr Białowolski \\ Janusz Czapiński \\ Izabela Grabowska \\ Irena E. Kotowska \\ Tomasz Panek \\ Paweł Strzelecki \\ Antoni Sułek \\ Tadeusz Szumlicz \\ Dorota Węziak-Białowolska
}

\section{Main sponsors of the project \\ Ministry of Labour and Social Policy \\ National Bank of Poland}

\section{Other sponsors}

University of Finance and Management in Warsaw

Ministry of Science and Higher Education

Telekomunikacja Polska SA

Centertel

Bank Zachodni WBK

BRE Bank SA

Chief Sanitary Inspector

\section{Media sponsorship}

The PIIIIIA.PI weekly

Radio TOK FM

Research carried out by: the Polish Statistical Association

Organisational and financial project management: Office for Statistical Analyses and Research of the Polish Statistical Association and the University of Finance and Management in Warsaw

Interviewers: Central Statistical Office

Result tables (Annexes 1, 2, 3 and 4) prepared by: Tomasz Jerzyński, Janusz Czapiński and Elżbieta Panek

Copyright (C) 2011 by the Council for Social Monitoring

Although financed from both private and public resources- the current edition from the Human Capital Operational Programme (from ESF funds), a research grant from the Ministry of Science and Higher Education and funds from the National Bank of Poland - the Social Diagnosis study is an entirely public undertaking. The complete set of tables with response distribution* and a database of cumulated data from six waves (of 2000, 2003, 2005, 2007, 2009, and 2011) is available free of charge at the website www.diagnoza.com.

\footnotetext{
* In all sets of response distribution tables, the order of variables from the questionnaires has been preserved.
} 
Please use the following citation when referring to this chapter: Czapiński, J. (2011). Summary. Social Diagnosis 2011. Objective and Subjective Quality of Life in Poland. [Special issue]. Contemporary Economics, 5(3), 262-285 DOI: 10.5709/ce.1897-9254.61

\section{SUMMARY}

Janusz Czapiński

\subsection{A plus for the Pole, a minus for the Poles}

We still live in a culture of envy and distrust, and we have not yet embarked on the journey towards civil society. But we have been developing, and changes have been taking place at a fairly good pace even in these times of worldwide economic crisis, though over recent years the process has been much faster at the individual rather than collective level. This is clearly visible when we compare the financial situation of Polish families with the condition of the central budget; between 2008 and 2010, similarly as in previous years, the Poles have been getting richer at a pace similar to the growth of $\mathrm{GDP}^{69}$, while the State; i.e. the central budget, has been getting poorer at a dramatic pace in recent years (Figure 9.1.1). Starting with the middle of the previous decade, budget income grew faster than the GDP and household income, mainly thanks to EU grants and loans. The latter inflated public debt, bringing it dangerously close to the first prudential threshold specified in the Constitution. A fierce public debate over that issue unfolded and still is unfolding. In the light of the uncertain economic situation in the world and in our closest European surroundings, the tone of the debate has become increasingly forboding, if not to say catastrophic. This however, has not impressed our fellow citizens to a great extent. They have not lost their optimism even though the growth rate of affluence has dropped dramatically and a majority of well-being indicators continue to grow as in the best times of the economic boom.

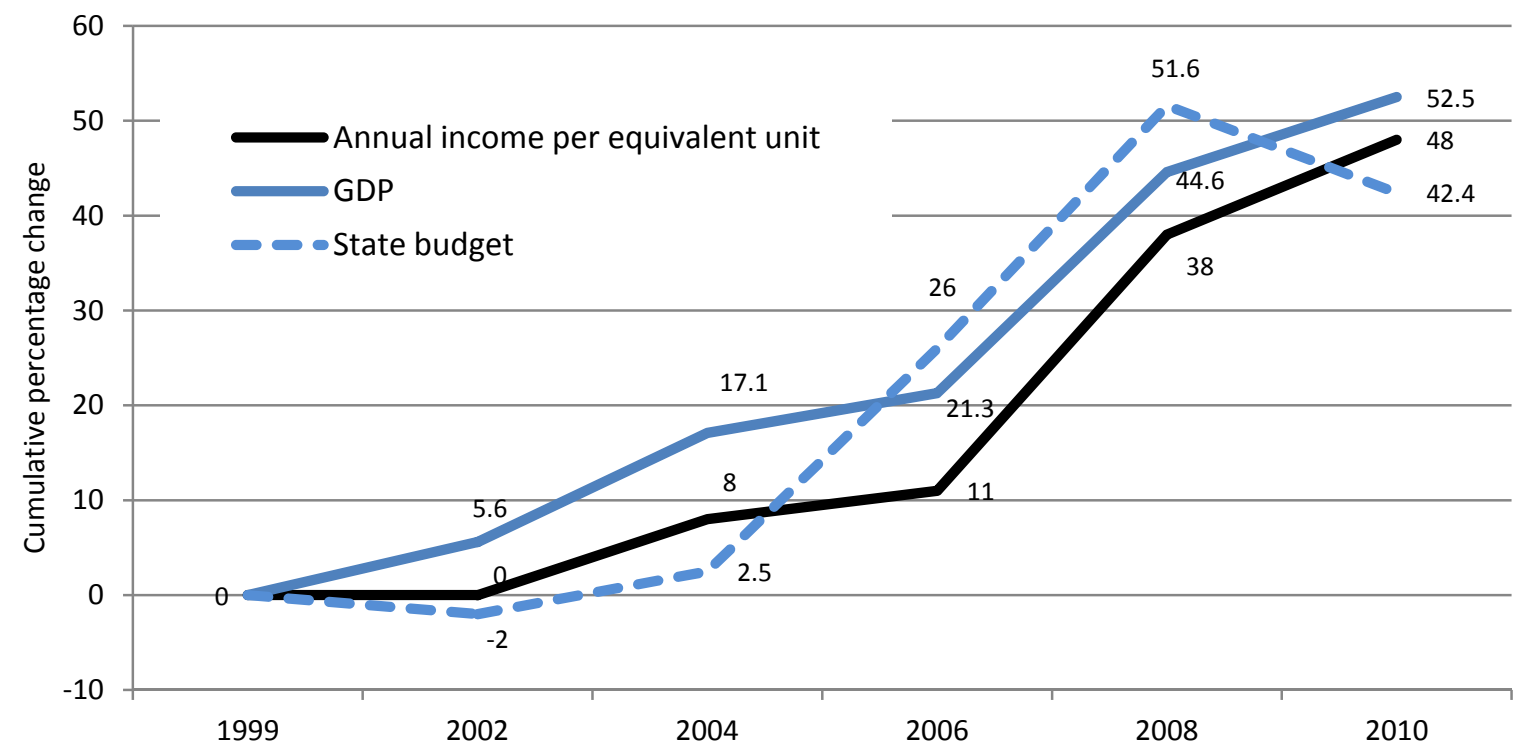

Figure 9.1.1. Cumulative percentage change in real values of annual household per capita income, the GDP and the State budget between 1999 and 2010

The Poles have become fairly good at playing with the State and see less and less connection between what the authorities do and what their lives look like.

The resourcefulness of the Poles helps them improve their own existence without concern for anyone else or the condition of the wider community. The deepening rupture between citizens and their State is best illustrated by the contrast between the evaluation of the domestic situation and the percentage of respondents who live in households with income insufficient for satisfying ongoing needs (Table 9.1.1).

\footnotetext{
${ }^{69}$ The data concern monthly income per equivalent unit from the year before the survey; i.e. 2010 in the case of the 2011 survey. A comparison of income from the month prior to the survey (March-April in 2011) does not reveal any such growth between 2009 and 2011 (cf. section 4.1).
} 
Even though existence at the individual level has been improving systematically (the number of poor households has dropped nearly three times since 1992), we remain dissatisfied with the situation in the country (the level of satisfaction nearly the same as in 1997. Consistently since the beginning of the transition this has been the lowest indicator of satisfaction in a set of approx. twenty various aspects of life - cf. section 5.2).

This perfectly illustrates the progress the Poles have made in enhancing their quality of life and progress made by Poland in the eyes of its citizens. We are still developing in a molecular manner rather than collectively (Czapiński, 2008). Presumably, the fundamental reason for that is the lack of social capital (Czapiński, 2011b).

Table 9.1.1. Percentage of households that declare their regular income as insufficient to meet their ongoing needs and the percentage of adult Poles satisfied with the situation in the country between 1992 and 2011

\begin{tabular}{|c|c|c|c|c|c|c|c|c|c|c|c|c|}
\hline Indicator & 1992 & 1993 & 1994 & 1995 & 1996 & 1997 & 2000 & 2003 & 2005 & 2007 & 2009 & 2011 \\
\hline $\begin{array}{l}\text { Percentage of } \\
\text { households with } \\
\text { regular income } \\
\text { insufficient to } \\
\text { meet their needs }\end{array}$ & 70.6 & 74.2 & 68.8 & 64.5 & 64.8 & 66.2 & 46.7 & 42.3 & 37.0 & 30.2 & 28.0 & 25.7 \\
\hline $\begin{array}{l}\text { Percentage of } \\
\text { those satisfied } \\
\text { with the situation } \\
\text { in the country }\end{array}$ & 9.4 & 8.2 & 11.2 & 16.4 & 20.1 & 25.7 & 19.7 & 14.1 & 12.6 & 19.3 & 27.0 & 26.0 \\
\hline
\end{tabular}

Growing individual resourcefulness is not matched by an increase in the ability to cooperate (cf. section 6.3). We are not learning to cooperate because we generally do not trust each other; we only make an exception for family members and less often for neighbours. We also do not trust institutions in general (with the exception of the NBP) (Figure 9.1.2). 


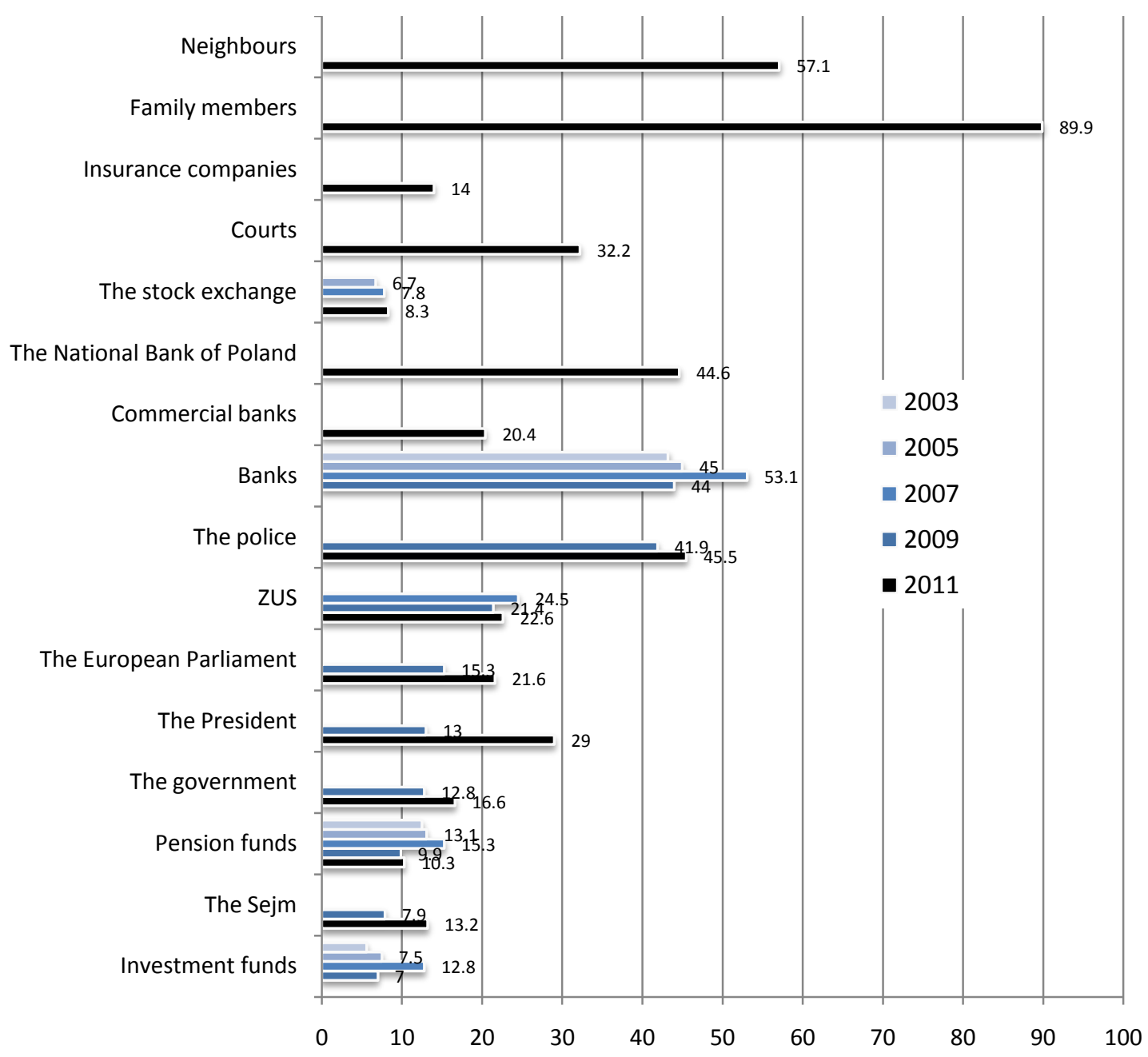

NOTE: The results of the European Social Survey of 2006/8 regarding the European Parliament and the Polish Sejm are fairly similar when looking at respondents who selected answers 7 to 10 at a 10-point scale, with 10 defined as "absolute confidence". 5\% trust the national parliament and $16.4 \%$ trust the European Parliament.

\section{Figure 9.1.2. Percentage of respondents who declare confidence in various persons and institutions}

Poland, after Bulgaria, is an EU Member State where the difference between confidence in the European Parliament and in the national legislative body is the greatest in favour of the former. ${ }^{70}$ It is also alarming that in social groups which have or will have a decisive influence on the development of the country; i.e. among residents of large agglomerations, young people and the well-educated, the hiatus between confidence in national and European authorities is the greatest (Figure 9.1.3). If we want to - and in our opinion we should - develop collectively, we urgently need to introduce a special subject, provisionally called civil skills in schools and perhaps even already in kindergartens. Young Poles have a fairly good knowledge of society and in this respect they win international rankings, but at the same time they are last in the same rankings in terms of applying civil knowledge in practice. They do not know how to get organised, to cooperate, they do not get involved in volunteer activities and they are as "molecular" as their parents (see www.szkolabezprzemocy.pl). Thus, they do not need classes in the standard lectureand-textbook form, but rather such forms of education (or actually upbringing) that will show them the real benefits that come with "taking the risk" of cooperation. Without serious investments in social capital we may forget the dreams of thousands of kilometres of motorways.

Apart from schools, there are also two other milieus where one could successfully persuade fellow citizens to trust more and to cooperate; i.e. public administration offices and enterprises. Regarding offices, legal regulations and the culture of officials are the key issues. The regulations followed by officers are

\footnotetext{
${ }^{70}$ In all new EU Member States citizens place more confidence in the European Parliament rather than in the national assembly, as opposed to "old" EU countries.
} 
designed to counter potential fraud, making it impossible to stop the vicious circle of distrust. Obviously, many enterprises appreciate the value of social capital, yet most do not know how to build it. This calls for training advisors and trainers in this particular respect, HR on its own will not suffice.

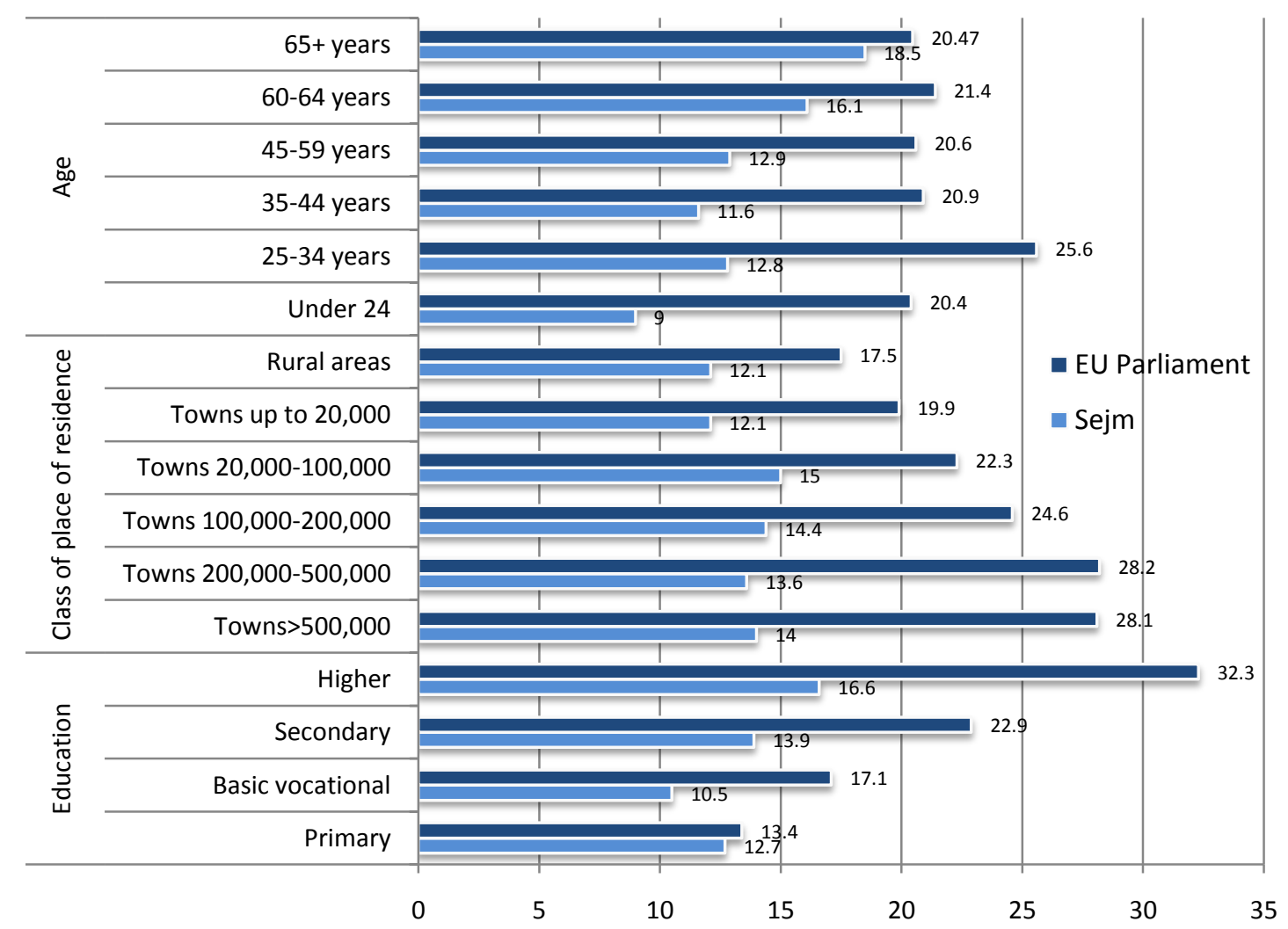

Figure 9.1.3. Confidence in the Sejm of the Republic of Poland and in the European Parliament in various social and demographic groups 


\subsection{The quality of life of various socio-demographic groups}

It is worth concluding with one general question: how varied are Poles' living conditions and their quality of life today and how has this variation changed in the past two years? Is society becoming more deeply or less stratified? For who is life easier and for who is it more difficult? Are the weak becoming even weaker and the strong even stronger?

Let us see how the multi-dimensional quality of life, which covers the most important indicators discussed separately in the chapters above, stratifies Polish society today. Can we speak of straightforward winners and losers, how big are the differences between them, and are these differences getting bigger or smaller in different dimensions of the quality of life?

When designing synthetic indicators of the quality of life, we endeavoured to strike a balance between objective and subjective indicators, as well as to take possibly the widest spectrum of various aspects into account. We distinguished eight dimensions assumed to cover independent content areas, which served to build up a general synthetic indicator of the quality of life:

- social capital - activity for the benefit of the local community, participation in self-government elections in 2010 (participation in parliamentary elections in 2007 was considered in the 2009 survey, participation in self-government elections was included in the 2007 one, and participation in the EU referendum was studied in 2005), participation in non-obligatory meetings, positive attitude to democracy, membership in organisations and serving functions in them, the belief that most people can be trusted;

- psychological well-being - sense of happiness, assessment of life-as-a-whole, incidence of mental depression symptoms, assessment of the past year;

- physical well-being - incidence of somatic symptoms, serious disease in the past year, degree of disability, intensity of health-related stress;

- social well-being - lack of the feeling of loneliness, a sense of being loved and respected, number of friends;

- civilisation level - educational level, ownership of modern communication devices and familiarity with them (satellite or cable TV, laptop, desktop computer, mobile phone, Internet connection, computer skills, Internet use), active command of foreign languages, driving license;

- material well-being - household income per equivalent unit, number of goods and appliances owned, ranging from automatic washing-machine to a motorboat or summer house (excluding appliances included in the civilisation level indicator);

- stress in life - a sum of six categories of stress measured by experiences related to finance, work, liaison with public administration offices, bringing up children, the marriage relationship, environmental protection (home, surroundings);

- pathology - alcohol abuse and drug use, smoking, consulting a psychiatrist or psychologist, being a criminal or victim of crime (burglaries, assaults, thefts).

Each partial indicator was a sum of standardised component variables, each of the latter measured on a different scale. Partial indicators were then standardized themselves and the sum of their standardized values formed the general indicator of the quality of life, which in turn was also standardized at the end. In such a form, these indicators are relative in nature and only show the position of particular groups and individuals in relation to the average of the sample.

Before we go on to discuss social differences in the general indicator of the quality of life, let us see to what extent partial indicators correlate with one another, whether they form one coherent syndrome or whether similarly to exclusion indicators they constitute several relatively independent factors which make it possible for individuals and social groups to compensate for shortages in one area with a better position in other areas.

Factor analysis with varimax rotation reveals two independent explanatory factors in four waves, which together explain a total of approx. 50\% of variance in partial indicators (Table 9.2.1). The first factor, which explains the greatest proportion of variance (approx. 30\%), may be described as civilisation-related living conditions (shortened to living conditions); these are mainly defined by the civilisation level and material well-being, but they also include social capital, physical well-being and psychological well-being. The other factor, which explains $18 \%$ to $19 \%$ of variation, is lifestyle mainly defined by stress in life, social wellbeing and pathology. It shares two aspects with the category of living conditions, namely psychological well-being and physical well-being. Thus, (mental and physical) health is determined both by living conditions and by lifestyle. 
This pattern of results confirms the statement that there is no single dimension of the quality of life in Poland at present. Thus, the less well-off are not very modern and show little social activity, but may nevertheless enjoy other favours of fate: absence of pathology, little stress and considerable social support.

Table 9.2.1. Results of factor analysis with varimax rotation for aspects of the quality of life

\begin{tabular}{|c|c|c|c|c|c|c|c|c|}
\hline \multirow{3}{*}{ Aspects of the quality of life } & \multicolumn{8}{|c|}{ Factor loadings } \\
\hline & \multicolumn{4}{|c|}{ Civilisation level } & \multicolumn{4}{|c|}{ Lifestyle } \\
\hline & 2011 & 2009 & 2007 & 2005 & 2011 & 2009 & 2007 & 2005 \\
\hline Civilisation level & 0.848 & 0.851 & 0.845 & 0.832 & & & & \\
\hline Material well-being & 0.747 & 0.742 & 0.720 & 0.722 & & & & \\
\hline Social capital & 0.461 & 0.497 & 0.481 & 0.528 & & & & \\
\hline Physical well-being & 0.492 & 0.508 & 0.514 & 0.448 & 0.449 & 0.441 & 0.422 & 0.481 \\
\hline Psychological well-being & 0.604 & 0.619 & 0.609 & 0.560 & 0.607 & 0.592 & 0.599 & 0.653 \\
\hline Social well-being & & & & & 0.577 & 0.595 & 0.628 & 0.612 \\
\hline Life stress & & & & & 0.690 & 0.659 & 0.673 & 0.643 \\
\hline Pathologies & & & & & 0.546 & 0.547 & 0.524 & 0.559 \\
\hline Percentage of variance explained & 31.2 & 31.8 & 30.4 & 29.8 & 18.7 & 18.2 & 18.4 & 19.4 \\
\hline
\end{tabular}

NOTE: the table shows factor loadings with values in excess of 0.4

Table 9.2.2. General indicator of the quality of life in entire samples between 2005 and 2011 in cross-section by socio-demographic group

\begin{tabular}{|c|c|c|c|c|c|c|c|c|}
\hline \multicolumn{4}{|c|}{ Rank } & \multirow{2}{*}{ Socio-demographic group } & \multicolumn{4}{|c|}{ Quality of life } \\
\hline 2011 & 2009 & 2007 & 2005 & & 2011 & 2009 & 2007 & 2005 \\
\hline 1 & 1 & 1 & 2 & Higher and post-secondary & 0.60 & 0.65 & 0.65 & 0.62 \\
\hline 2 & 2 & 2 & 1 & School and university students & 0.56 & 0.53 & 0.56 & 0.63 \\
\hline 3 & 3 & 3 & 4 & Private entrepreneurs & 0.48 & 0.50 & 0.49 & 0.45 \\
\hline 4 & 5 & 5 & 3 & Those aged $18-24$ years & 0.43 & 0.41 & 0.40 & 0.48 \\
\hline 5 & 4 & 4 & 5 & Public sector employees & 0.41 & 0.46 & 0.44 & 0.44 \\
\hline 6 & 6 & 6 & 7 & Those aged $25-34$ years & 0.35 & 0.38 & 0.33 & 0.30 \\
\hline 7 & 7 & 9 & 13 & Towns with 500,000 and more inhabitants & 0.31 & 0.28 & 0.20 & 0.11 \\
\hline 8 & 8 & 8 & 9 & Married couples with 2 children & 0.27 & 0.25 & 0.24 & 0.21 \\
\hline 9 & 10 & 7 & 6 & Unmarried men/women & 0.22 & 0.22 & 0.26 & 0.32 \\
\hline 10 & 9 & 12 & 8 & Private sector employees & 0.18 & 0.25 & 0.11 & 0.21 \\
\hline 11 & 11 & 11 & 12 & Married couples with 1 child & 0.17 & 0.22 & 0.15 & 0.13 \\
\hline 12 & 13 & 19 & 21 & Those aged $35-44$ years & 0.16 & 0.15 & 0.03 & -0.03 \\
\hline 13 & 12 & 10 & 10 & Secondary education & 0.12 & 0.18 & 0.17 & 0.20 \\
\hline 14 & 14 & 17 & 17 & Married couples with 3 and more children & 0.08 & 0.10 & 0.03 & 0.03 \\
\hline 15 & 15 & 18 & 20 & Married men/women & 0.08 & 0.09 & 0.03 & -0.01 \\
\hline 16 & 16 & 14 & 15 & Towns with $200,000-500,000$ inhabitants & 0.06 & 0.08 & 0.07 & 0.09 \\
\hline 17 & 17 & 15 & 14 & Men & 0.06 & 0.07 & 0.06 & 0.09 \\
\hline 18 & 21 & 21 & 19 & Multi-family households & 0.02 & -0.02 & 0.01 & 0.00 \\
\hline 19 & 22 & 16 & 16 & Towns with fewer than 20,000 inhabitants & 0.00 & -0.03 & 0.03 & 0.09 \\
\hline 20 & 20 & 22 & 18 & Married couples with no children & -0.01 & 0.01 & -0.05 & 0.02 \\
\hline 21 & 19 & 20 & 22 & Towns with $20,000-100,000$ inhabitants & -0.02 & 0.04 & 0.02 & -0.06 \\
\hline 22 & 18 & 13 & 11 & Towns with $100,000-200,000$ inhabitants & -0.04 & 0.04 & 0.10 & 0.18 \\
\hline 23 & 23 & 23 & 23 & Women & -0.05 & -0.06 & -0.05 & -0.07 \\
\hline 24 & 25 & 24 & 26 & Farmers & -0.05 & -0.11 & -0.06 & -0.13 \\
\hline 25 & 24 & 33 & 36 & Non-family multi-person households & -0.09 & -0.09 & -0.40 & -0.63 \\
\hline 26 & 26 & 25 & 24 & Rural areas & -0.10 & -0.12 & -0.12 & -0.09 \\
\hline 27 & 29 & 28 & 28 & Other professionally inactive & -0.15 & -0.19 & -0.18 & -0.21 \\
\hline 28 & 27 & 27 & 27 & Those aged $45-59$ years & -0.15 & -0.15 & -0.16 & -0.16 \\
\hline 29 & 28 & 26 & 25 & Basic vocational education & -0.16 & -0.17 & -0.14 & -0.11 \\
\hline 30 & 30 & 29 & 29 & Those aged $60-64$ years & -0.24 & -0.27 & -0.21 & -0.28 \\
\hline 31 & 32 & 30 & 30 & Single-parent families & -0.27 & -0.36 & -0.30 & -0.30 \\
\hline 32 & 31 & 32 & 32 & Unemployed persons & -0.33 & -0.35 & -0.40 & -0.35 \\
\hline 33 & 33 & 31 & 31 & Retirees & -0.39 & -0.39 & -0.33 & -0.31 \\
\hline 34 & 35 & 34 & 34 & Divorced men/women & -0.52 & -0.56 & -0.44 & -0.52 \\
\hline 35 & 34 & 36 & 35 & Non-family one-person households & -0.57 & -0.53 & -0.58 & -0.56 \\
\hline 36 & 36 & 35 & 33 & Those aged $65+$ years & -0.61 & -0.62 & -0.53 & -0.50 \\
\hline 37 & 37 & 37 & 38 & Widower/widow & -0.72 & -0.73 & -0.71 & -0.69 \\
\hline 38 & 39 & 38 & 37 & Primary education & -0.86 & -0.85 & -0.76 & -0.68 \\
\hline 39 & 38 & 39 & 39 & Pensioners & -0.88 & -0.82 & -0.77 & -0.80 \\
\hline
\end{tabular}

However, the above-mentioned independence of two factors of the quality of life at the level of the individual; i.e. in respect of individuals, may disappear or radically diminish in cross-section by sociodemographic group. It is not impossible that some segments of society suffer, like the biblical Job, all possible calamities while others enjoy the good life in all its aspects. In order to see whether this indeed is the case, we specified the position on the scale of one general and eight specific aspects of the quality of life of 174 groups determined by a range of not fully separable demographic and social criteria such as age, 
gender, educational level, class of place of residence, voivodeship, subregion, town, family type, social and professional status, occupation as currently pursued and marital status. The results are presented in Tables 9.2.2 to 9.2.9 (general indicator of the quality of life in 2011, 2009, 2007 and 2005) and in Tables 1-8 in Annex 6 (partial indicators of the quality of life in 2011).

Despite the fact that particular groups have different positions in respect of individual partial aspects, the general indicator of the quality of life clearly shows for whom life is good at present in Poland and for whom it is difficult, who has recently experienced an improvement and for whom there has been a deterioration. Undoubted beneficiaries include those with higher education, young people, entrepreneurs, residents of the largest cities (e.g. of Warsaw, Poznań, Kraków, Szczecin, Trójmiasto), the Mazowieckie, Małopolskie, Pomorskie and Wielkopolskie Voivodeships, the Tyski, Warszawski Zachodni, Poznański, Bydgosko-Toruński and Łódzki subregions, university teachers, doctors, IT specialists, managers, senior officials and lawyers. The poorest quality of life is definitely experienced by pensioners those with primary education, widowed persons, the elderly (aged 65 years and above), those who live on their own, divorcees, retirees and unemployed persons, residents of the Świętokrzyskie, Lubuskie and Lubelskie Voivodeships, of Radom, Kielce, Bielsko Biała, the Sieradzki, Sandomiersko-Jędrzejowski and Radomski subregions, farmers who produce for their own needs only, housekeepers and cleaners and unskilled workers.

Table 9.2.3. General indicator of the quality of life in the 2009-2011 panel sample in crosssection by socio-demographic group

\begin{tabular}{|c|c|c|c|c|c|c|c|c|}
\hline \multicolumn{2}{|c|}{ Rank } & \multirow[b]{2}{*}{ Socio-demographic group } & \multicolumn{3}{|c|}{2011} & \multicolumn{3}{|c|}{2009} \\
\hline 2011 & 2009 & & Average & $\begin{array}{l}\text { Standard } \\
\text { deviation }\end{array}$ & $\mathrm{N}$ & Average & $\begin{array}{l}\text { Standard } \\
\text { deviation }\end{array}$ & $\mathrm{N}$ \\
\hline 1 & 2 & School and university students & 0.67 & 0.74 & 1001 & 0.59 & 0.79 & 884 \\
\hline 2 & 1 & Higher education & 0.63 & 0.86 & 2726 & 0.66 & 0.86 & 2520 \\
\hline 3 & 4 & Those aged $18-24$ years & 0.53 & 0.79 & 1380 & 0.47 & 0.80 & 1390 \\
\hline 4 & 3 & Private entrepreneurs & 0.52 & 0.91 & 483 & 0.56 & 0.85 & 464 \\
\hline 5 & 5 & Public sector employees & 0.44 & 0.86 & 1644 & 0.45 & 0.89 & 1827 \\
\hline 6 & 6 & Those aged $25-34$ years & 0.38 & 0.85 & 1982 & 0.41 & 0.86 & 2062 \\
\hline 7 & 8 & Married couples with 2 children & 0.30 & 0.89 & 2593 & 0.26 & 0.92 & 2510 \\
\hline 8 & 10 & Unmarried men/women & 0.25 & 1.01 & 2932 & 0.23 & 1.00 & 2702 \\
\hline 9 & 7 & $\begin{array}{l}\text { Towns with 500,000 and more } \\
\text { inhabitants }\end{array}$ & 0.23 & 1.03 & 1513 & 0.27 & 1.04 & 1462 \\
\hline 10 & 9 & Private sector employees & 0.20 & 0.90 & 2898 & 0.25 & 0.90 & 2774 \\
\hline 11 & 11 & Married couples with 1 child & 0.20 & 0.98 & 2233 & 0.21 & 0.91 & 2420 \\
\hline 12 & 13 & Those aged $35-44$ years & 0.17 & 0.92 & 2017 & 0.15 & 0.91 & 2060 \\
\hline 13 & 16 & $\begin{array}{l}\text { Married couples with } 3 \text { and more } \\
\text { children }\end{array}$ & 0.17 & 0.91 & 1319 & 0.08 & 0.95 & 1266 \\
\hline 14 & 12 & Secondary education & 0.16 & 0.89 & 3982 & 0.18 & 0.89 & 3863 \\
\hline 15 & 14 & Towns with $200,000-500,000$ inhabitants & 0.09 & 1.01 & 1238 & 0.09 & 1.01 & 1182 \\
\hline 16 & 17 & Married men/women & 0.09 & 0.92 & 7469 & 0.08 & 0.94 & 7727 \\
\hline 17 & 15 & Men & 0.07 & 1.01 & 5729 & 0.08 & 1.01 & 5668 \\
\hline 18 & 18 & Towns with $20,000-100,000$ inhabitants & 0.01 & 0.99 & 2441 & 0.04 & 0.97 & 2449 \\
\hline 19 & 19 & Towns with $100,000-200,000$ inhabitants & 0.00 & 0.97 & 922 & 0.03 & 0.96 & 908 \\
\hline 20 & 20 & Multi-family & 0.00 & 0.88 & 1592 & 0.03 & 0.86 & 1425 \\
\hline 21 & 26 & Farmers & -0.02 & 0.79 & 559 & -0.12 & 0.92 & 602 \\
\hline 22 & 21 & $\begin{array}{l}\text { Towns with fewer than } 20,000 \\
\text { inhabitants }\end{array}$ & -0.03 & 1.02 & 1569 & -0.02 & 1.02 & 1565 \\
\hline 23 & 22 & Married couples with no children & -0.04 & 0.96 & 1982 & -0.04 & 1.01 & 2122 \\
\hline 24 & 23 & Women & -0.06 & 0.99 & 6917 & -0.07 & 0.99 & 6819 \\
\hline 25 & 24 & Those aged $45-59$ years & -0.07 & 1.02 & 3707 & -0.10 & 1.05 & 3862 \\
\hline 26 & 25 & Rural areas & -0.09 & 0.98 & 4962 & -0.12 & 0.98 & 4922 \\
\hline 27 & 27 & Other professionally inactive & -0.11 & 1.02 & 1012 & -0.13 & 1.00 & 1197 \\
\hline 28 & 28 & Basic vocational education & -0.15 & 0.90 & 3781 & -0.14 & 0.89 & 3907 \\
\hline 29 & 29 & Those aged $60-64$ years & -0.18 & 0.99 & 1072 & -0.21 & 0.99 & 917 \\
\hline 30 & 33 & Single-parent families & -0.26 & 0.98 & 1146 & -0.35 & 1.03 & 1128 \\
\hline 31 & 30 & Non-family multi-person & -0.27 & 1.11 & 75 & -0.22 & 1.09 & 76 \\
\hline 32 & 32 & Unemployed persons & -0.31 & 1.07 & 774 & -0.35 & 0.97 & 721 \\
\hline 33 & 31 & Retirees & -0.32 & 0.94 & 3139 & -0.33 & 0.92 & 2962 \\
\hline 34 & 34 & Divorced men/women & -0.47 & 1.14 & 655 & -0.47 & 1.15 & 620 \\
\hline 35 & 36 & Those aged $65+$ years & -0.56 & 0.93 & 2487 & -0.55 & 0.90 & 2196 \\
\hline 36 & 35 & Non-family one-person & -0.61 & 1.09 & 1705 & -0.53 & 1.10 & 1541 \\
\hline 37 & 37 & Widower/widow & -0.67 & 0.94 & 1554 & -0.67 & 0.93 & 1438 \\
\hline 38 & 38 & Pensioners & -0.83 & 0.90 & 1003 & -0.80 & 0.96 & 1026 \\
\hline 39 & 39 & Primary education & -0.83 & 0.88 & 2142 & -0.82 & 0.89 & 2195 \\
\hline
\end{tabular}

However, the question arises as to how durable these differences are. Do they remain the same, or are they growing or perhaps diminishing? A comparison of data from the four and last two measurements proves that the ranking of quality of life is essentially stable. Few groups have changed their position to an 
extent that could be deemed statistically significant. Over the past 6 years the quality of life improved for residents of the largest cities and those aged 25-34 and deteriorated for the eldest and those with the lowest level of education. In cross-section by town and voivodeship the changes were relatively small, only the quality of life of the residents of Torun increased as compared to 2007, while that of the inhabitants of Gdynia decreased (although the latter are still the most satisfied with their city) ${ }^{71}$.

Table 9.2.4. General indicator of the quality of life in entire samples between 2005 and 2011 in cross-section by larger towns (absence of data means that in respect of the particular variable the town was represented by fewer than 60 respondents)

\begin{tabular}{|c|c|c|c|c|c|c|c|c|}
\hline \multicolumn{4}{|c|}{ Rank } & \multirow{2}{*}{ Town } & \multicolumn{4}{|c|}{ Quality of life } \\
\hline 2011 & 2009 & 2007 & 2005 & & 2011 & 2009 & 2007 & 2005 \\
\hline 2 & 2 & 8 & & Toruń & 0.40 & 0.42 & 0.14 & \\
\hline 4 & 5 & 6 & 4 & Kraków & 0.36 & 0.29 & 0.18 & 0.19 \\
\hline 5 & 3 & 1 & & Gdynia & 0.25 & 0.34 & 0.50 & \\
\hline 6 & 9 & 10 & 8 & Szczecin & 0.23 & 0.22 & 0.02 & 0.05 \\
\hline 9 & 6 & 2 & 1 & Gdańsk & 0.11 & 0.25 & 0.27 & 0.27 \\
\hline 10 & 14 & 12 & 10 & Łódź & 0.09 & -0.03 & 0.00 & -0.08 \\
\hline 11 & 10 & 15 & 6 & Lublin & -0.01 & 0.17 & -0.15 & 0.10 \\
\hline 12 & 12 & 9 & & Częstochowa & -0.02 & 0.08 & 0.03 & \\
\hline 13 & 15 & 11 & 7 & Katowice & -0.03 & -0.06 & 0.00 & 0.09 \\
\hline 18 & 16 & 13 & & Wałbrzych & -0.16 & -0.08 & -0.07 & \\
\hline 19 & 18 & 16 & 9 & Kielce & -0.22 & -0.09 & -0.17 & -0.01 \\
\hline 20 & 21 & & & Radom & -0.24 & -0.24 & -0.17 & \\
\hline
\end{tabular}

Table 9.2.5. General indicator of the quality of life in the 2009-2011 panel sample in crosssection by larger towns represented for particular variables by at least 60 respondents in each wave

\begin{tabular}{|c|c|c|c|c|c|c|c|c|}
\hline \multicolumn{2}{|c|}{ Rank } & \multirow{2}{*}{ Town } & \multicolumn{3}{|c|}{2011} & \multicolumn{3}{|c|}{2009} \\
\hline 2011 & 2009 & & Average & $\begin{array}{l}\text { Standard } \\
\text { deviation }\end{array}$ & $\mathrm{N}$ & Average & $\begin{array}{l}\text { Standard } \\
\text { deviation }\end{array}$ & $\mathrm{N}$ \\
\hline 1 & 2 & Poznań & 0.47 & 1.00 & 130 & 0.53 & 0.76 & 117 \\
\hline 2 & 1 & Toruń & 0.42 & 0.92 & 72 & 0.63 & 0.80 & 64 \\
\hline 3 & 3 & Warszawa & 0.37 & 1.10 & 500 & 0.46 & 1.05 & 502 \\
\hline 4 & 5 & Szczecin & 0.36 & 1.07 & 165 & 0.33 & 1.01 & 149 \\
\hline 5 & 7 & Gdynia & 0.34 & 0.98 & 69 & 0.23 & 0.96 & 70 \\
\hline 6 & 6 & Kraków & 0.28 & 0.90 & 295 & 0.24 & 1.03 & 281 \\
\hline 7 & 8 & Gdańsk & 0.24 & 1.09 & 128 & 0.18 & 1.13 & 171 \\
\hline 8 & 4 & Wrocław & 0.11 & 0.99 & 224 & 0.34 & 0.96 & 206 \\
\hline 9 & 11 & Jaworzno & 0.11 & 1.10 & 61 & 0.02 & 1.08 & 61 \\
\hline 10 & 10 & Bydgoszcz & 0.09 & 1.02 & 97 & 0.04 & 0.91 & 89 \\
\hline 11 & 13 & Sosnowiec & 0.07 & 0.89 & 71 & -0.01 & 0.97 & 85 \\
\hline 12 & 9 & Częstochowa & 0.03 & 0.75 & 101 & 0.12 & 0.89 & 79 \\
\hline 13 & 14 & Wałbrzych & 0.03 & 1.00 & 100 & -0.01 & 1.02 & 84 \\
\hline 14 & 16 & Białystok & 0.03 & 0.93 & 138 & -0.02 & 0.96 & 103 \\
\hline 15 & 18 & Lódź & -0.02 & 1.03 & 364 & -0.10 & 1.05 & 351 \\
\hline 16 & 15 & Katowice & -0.05 & 1.02 & 113 & -0.01 & 1.02 & 92 \\
\hline 17 & 17 & Lublin & -0.06 & 0.95 & 95 & -0.03 & 1.00 & 76 \\
\hline 18 & 12 & Bielsko-Biała & -0.11 & 0.90 & 88 & 0.01 & 1.20 & 99 \\
\hline 19 & 20 & Radom & -0.23 & 1.00 & 99 & -0.23 & 0.93 & 107 \\
\hline 20 & 19 & Kielce & -0.28 & 1.14 & 91 & -0.14 & 1.06 & 92 \\
\hline
\end{tabular}

\footnotetext{
${ }^{71}$ Changes between 2009 and 2011 in the panel sample in cross-section by town and voivodeship are definitely smaller and sometimes follow a different direction that the changes in entire samples.
} 
Table 9.2.6. General indicator of the quality of life between 2005 and 2011 in entire samples in cross-section by voivodeship

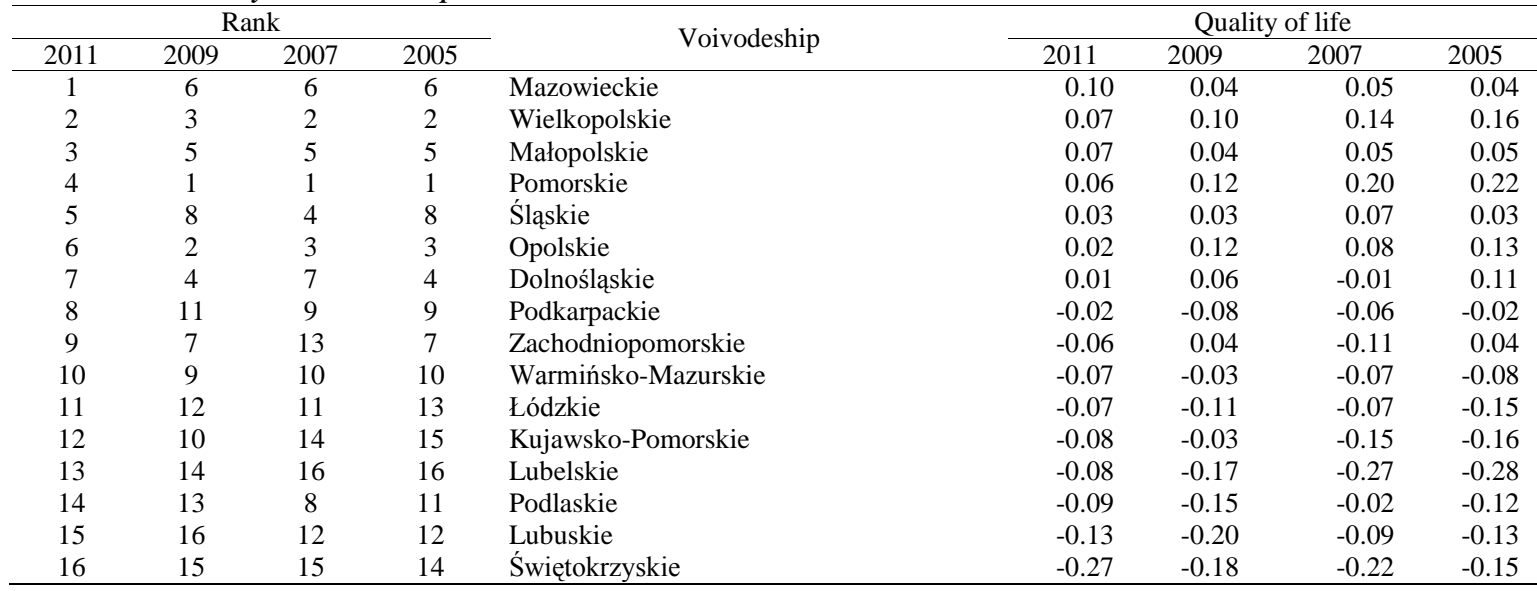

Table 9.2.7. General indicator of the quality of life in the panel sample between 2009 and 2011 in cross-section by voivodeship

\begin{tabular}{|c|c|c|c|c|c|c|c|c|}
\hline \multicolumn{2}{|c|}{ Rank } & \multirow[b]{2}{*}{ Voivodeship } & \multicolumn{3}{|c|}{2011} & \multicolumn{3}{|c|}{2009} \\
\hline 2011 & 2009 & & Average & $\begin{array}{l}\text { Standard } \\
\text { deviation }\end{array}$ & $\mathrm{N}$ & Average & $\begin{array}{l}\text { Standard } \\
\text { deviation }\end{array}$ & $\mathrm{N}$ \\
\hline 1 & 3 & Wielkopolskie & 0.15 & 0.96 & 956 & 0.10 & 0.94 & 1047 \\
\hline 2 & 5 & Pomorskie & 0.13 & 1.06 & 703 & 0.07 & 1.04 & 808 \\
\hline 3 & 6 & Małopolskie & 0.08 & 0.94 & 1122 & 0.06 & 1.02 & 1015 \\
\hline 4 & 8 & Mazowieckie & 0.05 & 1.05 & 1707 & 0.03 & 1.07 & 1755 \\
\hline 5 & 1 & Dolnośląskie & 0.03 & 0.96 & 928 & 0.10 & 0.96 & 891 \\
\hline 6 & 4 & Śląskie & 0.03 & 0.93 & 1459 & 0.08 & 0.96 & 1389 \\
\hline 7 & 7 & Zachodniopomorskie & 0.03 & 1.01 & 601 & 0.04 & 0.98 & 568 \\
\hline 8 & 2 & Opolskie & -0.03 & 0.96 & 368 & 0.10 & 0.97 & 334 \\
\hline 9 & 12 & Podkarpackie & -0.04 & 0.94 & 738 & -0.09 & 0.94 & 776 \\
\hline 10 & 10 & Warmińsko-Mazurskie & -0.06 & 1.00 & 478 & -0.05 & 0.99 & 507 \\
\hline 11 & 13 & Łódzkie & -0.07 & 0.95 & 970 & -0.10 & 0.98 & 965 \\
\hline 12 & 9 & Kujawsko-Pomorskie & -0.09 & 0.98 & 625 & -0.04 & 0.92 & 567 \\
\hline 13 & 11 & Podlaskie & -0.10 & 1.05 & 519 & -0.08 & 0.99 & 446 \\
\hline 14 & 15 & Lubelskie & -0.14 & 1.04 & 701 & -0.22 & 0.99 & 644 \\
\hline 15 & 16 & Lubuskie & -0.18 & 1.14 & 319 & -0.23 & 1.10 & 305 \\
\hline 16 & 14 & Świętokrzyskie & -0.25 & 1.12 & 451 & -0.14 & 1.07 & 471 \\
\hline
\end{tabular}


Table 9.2.8. Quality of life in entire samples of 2009 and 2011 in cross-section by subregion (NUTS3)

\begin{tabular}{|c|c|c|c|c|c|c|c|c|}
\hline \multicolumn{2}{|c|}{ Rank } & \multirow[b]{2}{*}{ Subregion } & \multicolumn{3}{|c|}{2011} & \multicolumn{3}{|c|}{2009} \\
\hline 2011 & 2009 & & Average & $\begin{array}{l}\text { Standard } \\
\text { deviation }\end{array}$ & $\mathrm{N}$ & Average & $\begin{array}{l}\text { Standard } \\
\text { deviation }\end{array}$ & $\mathrm{N}$ \\
\hline 1 & 1 & M. Warszawa & 0.46 & 1.02 & 913 & 0.42 & 1.09 & 893 \\
\hline 2 & 2 & M. Poznań & 0.36 & 0.99 & 207 & 0.32 & 0.88 & 235 \\
\hline 3 & 4 & M. Kraków & 0.36 & 0.93 & 486 & 0.29 & 0.97 & 449 \\
\hline 4 & 8 & Tyski & 0.25 & 0.98 & 200 & 0.20 & 1.02 & 201 \\
\hline 5 & 7 & M Szczecin & 0.23 & 1.04 & 261 & 0.22 & 1.01 & 237 \\
\hline 6 & 3 & Poznański & 0.19 & 1.01 & 238 & 0.32 & 0.92 & 186 \\
\hline 7 & 14 & Warszawski Zachodni & 0.19 & 0.95 & 315 & 0.13 & 1.09 & 332 \\
\hline 8 & 5 & Trójmiejski & 0.16 & 1.05 & 386 & 0.26 & 1.06 & 440 \\
\hline 9 & 12 & Rybnicki & 0.16 & 0.90 & 214 & 0.18 & 0.90 & 223 \\
\hline 10 & 13 & Bydgosko-Toruński & 0.15 & 0.96 & 415 & 0.16 & 0.95 & 331 \\
\hline 11 & 16 & Bytomski & 0.15 & 0.87 & 191 & 0.10 & 0.94 & 154 \\
\hline 12 & 23 & Łódzki & 0.15 & 0.88 & 159 & 0.04 & 1.11 & 174 \\
\hline 13 & 6 & M. Wrocław & 0.13 & 0.99 & 356 & 0.25 & 0.95 & 351 \\
\hline 14 & 9 & Legnicko-Głogowski & 0.11 & 0.85 & 232 & 0.20 & 0.93 & 194 \\
\hline 15 & 24 & Jeleniogórski & 0.10 & 0.95 & 368 & 0.04 & 0.94 & 364 \\
\hline 16 & 10 & Opolski & 0.09 & 0.94 & 353 & 0.20 & 0.95 & 319 \\
\hline 17 & 25 & Stupski & 0.09 & 1.04 & 232 & 0.04 & 1.08 & 296 \\
\hline 18 & 39 & M. Łódź & 0.09 & 0.98 & 538 & -0.03 & 1.04 & 432 \\
\hline 19 & 35 & Rzeszowski & 0.08 & 1.04 & 301 & -0.01 & 1.04 & 331 \\
\hline 20 & 19 & Warszawski Wschodni & 0.07 & 0.92 & 293 & 0.07 & 0.95 & 311 \\
\hline 21 & 26 & Elbląski & 0.07 & 0.99 & 298 & 0.03 & 1.08 & 249 \\
\hline 22 & 62 & Tarnowski & 0.07 & 0.92 & 299 & -0.28 & 1.13 & 225 \\
\hline 23 & 18 & Częstochowski & 0.04 & 0.91 & 401 & 0.08 & 0.90 & 281 \\
\hline 24 & 15 & Kaliski & 0.03 & 0.89 & 341 & 0.11 & 0.90 & 398 \\
\hline 25 & 27 & Bielski & 0.03 & 0.88 & 352 & 0.03 & 1.02 & 404 \\
\hline 26 & 17 & Pilski & 0.02 & 1.01 & 187 & 0.09 & 0.93 & 201 \\
\hline 27 & 21 & Wrocławski & 0.02 & 0.95 & 221 & 0.06 & 0.99 & 247 \\
\hline 28 & 49 & Leszczyński & 0.02 & 0.96 & 292 & -0.12 & 0.95 & 260 \\
\hline 29 & 31 & Nowosądecki & 0.01 & 0.85 & 416 & 0.00 & 0.97 & 339 \\
\hline 30 & 32 & Krośnieński & 0.00 & 0.94 & 342 & 0.00 & 0.87 & 347 \\
\hline 31 & 55 & Przemyski & 0.00 & 0.92 & 239 & -0.19 & 0.92 & 202 \\
\hline 32 & 28 & Starogardzki & -0.01 & 1.01 & 269 & 0.03 & 0.91 & 262 \\
\hline 33 & 29 & Lubelski & -0.01 & 1.02 & 349 & 0.01 & 0.97 & 310 \\
\hline 34 & 41 & Białostocki & -0.01 & 0.95 & 318 & -0.05 & 0.91 & 293 \\
\hline 35 & 22 & Sosnowiecki & -0.02 & 0.94 & 402 & 0.06 & 0.91 & 505 \\
\hline 36 & 20 & Gdański & -0.04 & 1.00 & 284 & 0.07 & 0.97 & 309 \\
\hline 37 & 44 & Skierniewicki & -0.04 & 0.91 & 186 & -0.08 & 0.91 & 179 \\
\hline 38 & 51 & Puławski & -0.04 & 0.96 & 255 & -0.17 & 0.97 & 213 \\
\hline 39 & 33 & Krakowski & -0.05 & 1.03 & 346 & 0.00 & 1.01 & 317 \\
\hline 40 & 56 & Gorzowski & -0.06 & 0.99 & 237 & -0.19 & 1.08 & 218 \\
\hline 41 & 34 & Nyski & -0.07 & 0.96 & 254 & 0.00 & 0.94 & 220 \\
\hline 42 & 37 & Gliwicki & -0.07 & 0.93 & 231 & -0.02 & 1.00 & 261 \\
\hline 43 & 45 & Katowicki & -0.08 & 1.03 & 410 & -0.09 & 0.99 & 364 \\
\hline 44 & 38 & Ełcki & -0.09 & 1.03 & 114 & -0.02 & 1.05 & 125 \\
\hline 45 & 47 & Ostrołęcko-Siedlecki & -0.09 & 0.96 & 420 & -0.10 & 0.95 & 490 \\
\hline 46 & 11 & Szczeciński & -0.10 & 1.00 & 130 & 0.20 & 0.94 & 120 \\
\hline 47 & 58 & Łomżyński & -0.11 & 1.20 & 242 & -0.21 & 1.10 & 228 \\
\hline 48 & 36 & Koniński & -0.12 & 0.98 & 281 & -0.01 & 0.96 & 380 \\
\hline 49 & 42 & Stargardzki & -0.13 & 0.97 & 227 & -0.07 & 0.88 & 194 \\
\hline 50 & 30 & Oświęcimski & -0.16 & 0.95 & 315 & 0.01 & 0.98 & 338 \\
\hline 51 & 60 & Chełmsko-Zamojski & -0.16 & 1.00 & 309 & -0.26 & 0.96 & 372 \\
\hline 52 & 52 & Tarnobrzeski & -0.17 & 0.94 & 255 & -0.18 & 0.99 & 305 \\
\hline 53 & 65 & Ciechanowsko-Płocki & -0.17 & 1.04 & 314 & -0.34 & 0.96 & 324 \\
\hline 54 & 64 & Bialski & -0.18 & 0.98 & 171 & -0.33 & 1.01 & 147 \\
\hline 55 & 43 & Koszaliński & -0.20 & 0.97 & 385 & -0.07 & 0.97 & 411 \\
\hline 56 & 53 & Włocławski & -0.20 & 0.95 & 417 & -0.18 & 0.90 & 398 \\
\hline 57 & 57 & Zielonogórski & -0.20 & 1.25 & 265 & -0.20 & 1.05 & 252 \\
\hline 58 & 48 & Kielecki & -0.21 & 1.21 & 366 & -0.11 & 1.08 & 378 \\
\hline 59 & 61 & Suwalski & -0.21 & 0.93 & 178 & -0.27 & 0.98 & 116 \\
\hline 60 & 46 & Olsztyński & -0.24 & 1.02 & 217 & -0.09 & 0.90 & 281 \\
\hline 61 & 40 & Grudziądzki & -0.27 & 0.99 & 227 & -0.03 & 0.89 & 216 \\
\hline 62 & 50 & Wałbrzyski & -0.27 & 1.03 & 399 & -0.14 & 1.03 & 426 \\
\hline 63 & 59 & Piotrkowski & -0.28 & 0.97 & 336 & -0.24 & 0.97 & 360 \\
\hline 64 & 66 & Radomski & -0.33 & 1.03 & 420 & -0.39 & 1.07 & 406 \\
\hline 65 & 54 & Sieradzki & -0.35 & 1.03 & 198 & -0.18 & 0.88 & 262 \\
\hline 66 & 63 & Sandomiersko-Jędrzejowski & -0.37 & 1.05 & 222 & -0.29 & 1.02 & 263 \\
\hline
\end{tabular}


272

Table 9.2.9. Quality of life in the 2009-2011 panel sample in the cross-section by subregion (NUTS3)

\begin{tabular}{|c|c|c|c|c|c|c|c|c|}
\hline \multicolumn{2}{|c|}{ Rank } & \multirow[b]{2}{*}{ Subregion } & \multicolumn{3}{|c|}{2011} & \multicolumn{3}{|c|}{2009} \\
\hline 2011 & 2009 & & Average & $\begin{array}{l}\text { Standard } \\
\text { deviation }\end{array}$ & $\mathrm{N}$ & Average & $\begin{array}{l}\text { Standard } \\
\text { deviation }\end{array}$ & $\mathrm{N}$ \\
\hline 1 & 1 & M. Poznań & 0.47 & 1.00 & 130 & 0.53 & 0.76 & 117 \\
\hline 2 & 2 & M. Warszawa & 0.37 & 1.10 & 500 & 0.45 & 1.05 & 505 \\
\hline 3 & 5 & M Szczecin & 0.36 & 1.07 & 165 & 0.33 & 1.01 & 149 \\
\hline 4 & 10 & Tyski & 0.34 & 0.92 & 129 & 0.23 & 0.91 & 102 \\
\hline 5 & 3 & Poznański & 0.31 & 1.00 & 114 & 0.45 & 1.02 & 108 \\
\hline 6 & 13 & Trójmiejski & 0.29 & 1.06 & 213 & 0.19 & 1.09 & 254 \\
\hline 7 & 9 & M. Kraków & 0.28 & 0.90 & 295 & 0.24 & 1.03 & 281 \\
\hline 8 & 15 & Warszawski Zachodni & 0.28 & 0.93 & 221 & 0.14 & 1.10 & 215 \\
\hline 9 & 24 & Łódzki & 0.27 & 0.84 & 115 & 0.05 & 1.15 & 121 \\
\hline 10 & 6 & Legnicko-Głogowski & 0.22 & 0.83 & 126 & 0.27 & 0.87 & 119 \\
\hline 11 & 11 & Rybnicki & 0.17 & 0.99 & 131 & 0.22 & 0.86 & 115 \\
\hline 12 & 37 & Słupski & 0.17 & 1.14 & 157 & -0.04 & 1.13 & 191 \\
\hline 13 & 25 & Szczeciński & 0.15 & 0.93 & 83 & 0.05 & 0.95 & 68 \\
\hline 14 & 7 & Warszawski Wschodni & 0.14 & 0.88 & 196 & 0.26 & 0.93 & 182 \\
\hline 15 & 36 & Leszczyński & 0.13 & 0.89 & 159 & -0.03 & 0.94 & 182 \\
\hline 16 & 14 & Bydgosko-Toruński & 0.12 & 1.02 & 205 & 0.17 & 0.99 & 191 \\
\hline 17 & 4 & M. Wrocław & 0.11 & 0.99 & 224 & 0.34 & 0.96 & 206 \\
\hline 18 & 26 & Krakowski & 0.11 & 1.00 & 253 & 0.03 & 1.06 & 237 \\
\hline 19 & 29 & Rzeszowski & 0.09 & 1.06 & 211 & 0.01 & 1.04 & 205 \\
\hline 20 & 40 & Tarnowski & 0.09 & 0.99 & 142 & -0.06 & 1.06 & 139 \\
\hline 21 & 17 & Bielski & 0.08 & 0.88 & 278 & 0.09 & 1.05 & 285 \\
\hline 22 & 21 & Białostocki & 0.08 & 0.93 & 246 & 0.07 & 0.88 & 207 \\
\hline 23 & 27 & Pilski & 0.07 & 0.92 & 122 & 0.03 & 0.84 & 135 \\
\hline 24 & 30 & Starogardzki & 0.07 & 0.82 & 149 & 0.01 & 0.84 & 174 \\
\hline 25 & 31 & Kaliski & 0.07 & 0.94 & 243 & 0.00 & 0.91 & 278 \\
\hline 26 & 35 & Wrocławski & 0.07 & 0.98 & 106 & -0.02 & 0.95 & 114 \\
\hline 27 & 22 & Elbląski & 0.06 & 1.00 & 212 & 0.07 & 1.04 & 208 \\
\hline 28 & 23 & Jeleniogórski & 0.05 & 0.92 & 240 & 0.06 & 0.94 & 225 \\
\hline 29 & 8 & Bytomski & 0.03 & 0.91 & 79 & 0.25 & 0.86 & 75 \\
\hline 30 & 12 & Opolski & 0.03 & 0.99 & 193 & 0.20 & 0.97 & 179 \\
\hline 31 & 16 & Częstochowski & 0.02 & 0.86 & 203 & 0.10 & 0.90 & 165 \\
\hline 32 & 20 & Sosnowiecki & 0.02 & 0.93 & 237 & 0.08 & 0.96 & 293 \\
\hline 33 & 28 & Krośnieński & -0.01 & 0.88 & 215 & 0.01 & 0.85 & 239 \\
\hline 34 & 34 & Koniński & -0.01 & 0.98 & 188 & -0.01 & 0.96 & 226 \\
\hline 35 & 42 & M. Łódź & -0.02 & 1.03 & 364 & -0.09 & 1.05 & 352 \\
\hline 36 & 59 & Puławski & -0.02 & 1.06 & 190 & -0.24 & 1.03 & 161 \\
\hline 37 & 19 & Gdański & -0.03 & 1.15 & 184 & 0.08 & 1.02 & 189 \\
\hline 38 & 46 & Nowosądecki & -0.04 & 0.89 & 221 & -0.11 & 0.95 & 173 \\
\hline 39 & 38 & Skierniewicki & -0.06 & 0.85 & 143 & -0.05 & 0.87 & 128 \\
\hline 40 & 43 & Ełcki & -0.06 & 1.02 & 75 & -0.09 & 1.05 & 75 \\
\hline 41 & 33 & Gliwicki & -0.07 & 0.85 & 148 & -0.01 & 0.91 & 155 \\
\hline 42 & 51 & Ostrołęcko-Siedlecki & -0.08 & 0.99 & 313 & -0.14 & 0.98 & 333 \\
\hline 43 & 60 & Gorzowski & -0.09 & 1.09 & 141 & -0.24 & 1.10 & 145 \\
\hline 44 & 32 & Nyski & -0.10 & 0.93 & 175 & -0.01 & 0.97 & 155 \\
\hline 45 & 39 & Stargardzki & -0.10 & 0.93 & 137 & -0.05 & 0.86 & 120 \\
\hline 46 & 18 & Oświęcimski & -0.12 & 0.91 & 211 & 0.08 & 0.96 & 184 \\
\hline 47 & 55 & Przemyski & -0.12 & 0.81 & 123 & -0.20 & 0.86 & 136 \\
\hline 48 & 49 & Katowicki & -0.14 & 1.04 & 254 & -0.12 & 0.97 & 201 \\
\hline 49 & 48 & Lubelski & -0.15 & 1.05 & 193 & -0.12 & 1.02 & 155 \\
\hline 50 & 61 & Tarnobrzeski & -0.15 & 0.92 & 189 & -0.25 & 0.97 & 196 \\
\hline 51 & 65 & Ciechanowsko-Płocki & -0.15 & 0.91 & 193 & -0.31 & 0.87 & 221 \\
\hline 52 & 41 & Grudziądzki & -0.16 & 0.91 & 144 & -0.07 & 0.89 & 118 \\
\hline 53 & 64 & Bialski & -0.17 & 0.97 & 115 & -0.27 & 0.96 & 101 \\
\hline 54 & 45 & Wałbrzyski & -0.19 & 1.01 & 233 & -0.11 & 0.97 & 228 \\
\hline 55 & 47 & Koszaliński & -0.19 & 0.97 & 216 & -0.11 & 0.99 & 230 \\
\hline 56 & 44 & Kielecki & -0.20 & 1.19 & 276 & -0.10 & 1.08 & 298 \\
\hline 57 & 53 & Olsztyński & -0.20 & 0.97 & 191 & -0.15 & 0.91 & 224 \\
\hline 58 & 50 & Sieradzki & -0.21 & 0.80 & 129 & -0.14 & 0.77 & 130 \\
\hline 59 & 62 & Chełmsko-Zamojski & -0.21 & 1.05 & 203 & -0.26 & 0.96 & 227 \\
\hline 60 & 54 & Włocławski & -0.22 & 0.97 & 275 & -0.18 & 0.86 & 258 \\
\hline 61 & 63 & Łomżyński & -0.24 & 1.19 & 167 & -0.26 & 1.09 & 139 \\
\hline 62 & 58 & Zielonogórski & -0.25 & 1.17 & 179 & -0.22 & 1.10 & 160 \\
\hline 63 & 52 & Suwalski & -0.26 & 1.03 & 105 & -0.15 & 1.02 & 99 \\
\hline 64 & 56 & Piotrkowski & -0.27 & 0.97 & 220 & -0.21 & 0.93 & 233 \\
\hline 65 & 57 & Sandomiersko-Jędrzejowski & -0.32 & 0.98 & 176 & -0.21 & 1.04 & 173 \\
\hline 66 & 66 & Radomski & -0.46 & 1.03 & 285 & -0.46 & 1.08 & 296 \\
\hline
\end{tabular}


The categories of respondents as defined by some of the criteria may differ only apparently in the sense that they are determined by some other criterion of division into groups correlated with a given group. Gender may serve as an example here. In all waves men score higher in terms of the value of the indicator of the quality of life. This, however, may result from the fact that women live longer and the quality of life deteriorates with age. Indeed, in the 2011 sample women's average life expectancy was more than 3 years longer than that of men while in the eldest group (65 years and above), where the quality of life is the worst, the proportion of women is nearly twice as large as that of men (63\% to 37\%). Results of the analysis of variance prove that indeed, the difference between men and women in respect of the quality of life is primarily determined by age (Figure 9.2.1). Only in the group of the eldest people is men's quality of life considerably better than that of women $^{72}$; in other age groups, with the exception of the 34-44 group, gender does not differentiate the quality of life.

The differentiating role of the age variable in respect of the quality of life may also be inflated due to the fact that in Poland there is a strong correlation between age and the level of education ${ }^{73}$, with the latter certainly important for the quality of life. The question therefore is whether the low quality of life of the elderly is only attributable to their age, or maybe also to the fact that on average they are much worse educated than younger people. It turns out that the indicator for determinative role of age in the regression analysis decreases nearly three times (from $13.1 \%$ to $4.6 \%$ of independently explained variance in the quality of life) when the equation is expanded to also include the level of education as well. The analysis of variance reveals a significant effect of interaction of age and educational level in respect of the quality of life (Figure 9.2.2). Higher education clearly mitigates the negative impact of age on the quality of life; the difference between those with better and poorer education in the eldest group is nearly four times as big as in the youngest group, which is mainly due to the fact that the quality of life changes considerably with age among those with poor education and virtually does not change among those with better education.

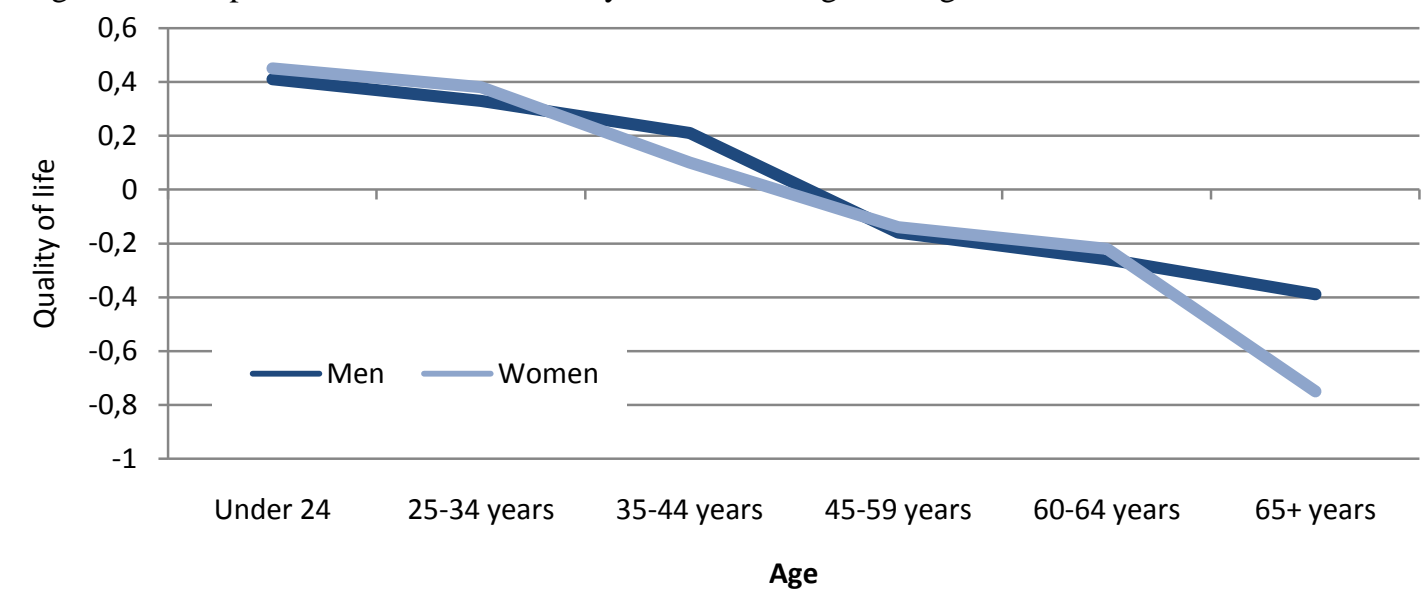

NOTE: main effect of age $F(5,19487)=510.132, p<0.000, \eta^{2}=0.116$; main effect of gender $F(1,19487)=2.132, p<0.01, \eta^{2}=0.001$; effect of interaction of age and gender $F(5,19487)=22.670, p<0.000, \eta^{2}=0.006$.

Figure 9.2.1. General indicator of the quality of life depending on age and gender

\footnotetext{
${ }^{72}$ Average age of women in this group is more than one year more than that of men.

${ }^{73}$ The correlation coefficient of age and educational level as measured by the number of years of schooling (together with those who have not completed education yet) amounts to -0.303 in the entire sample and to -0.441 in the sample of those who have already completed education .
} 


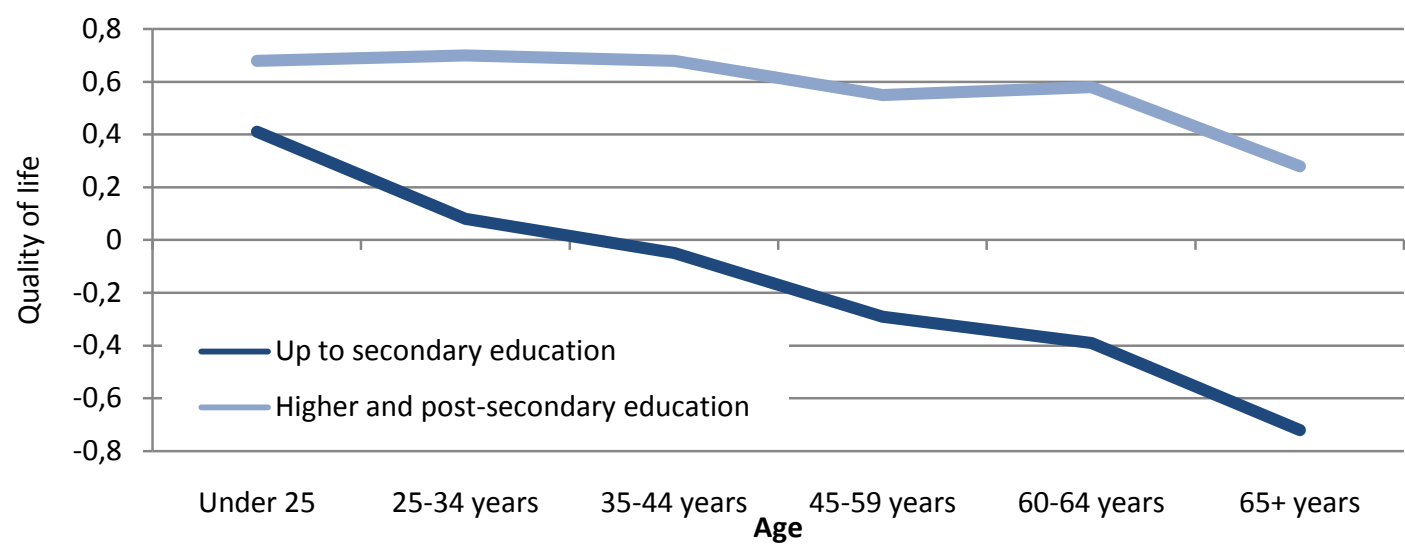

NOTE: main effect of age $F(5,19455)=135.907, p<0.000, \eta^{2}=0.034$; main effect of education $F(1,19455)=1382.491, p<0.000, \eta^{2}=$ 0.066; effect of interaction of age and education $F(5,19455)=24.336, p<0.000, \eta^{2}=0.006$.

Figure 9.2.2. General indicator of the quality of life depending on age and educational level, with gender control

Education also influences the differences in the quality of life of groups determined by social and professional status (Figure 9.2.3). Even though those with higher education live a better life in all groups, their predominance over those with lower education is not always the same. It is relatively small among entrepreneurs but enormous among employees, retirees, pensioners and unemployed persons.

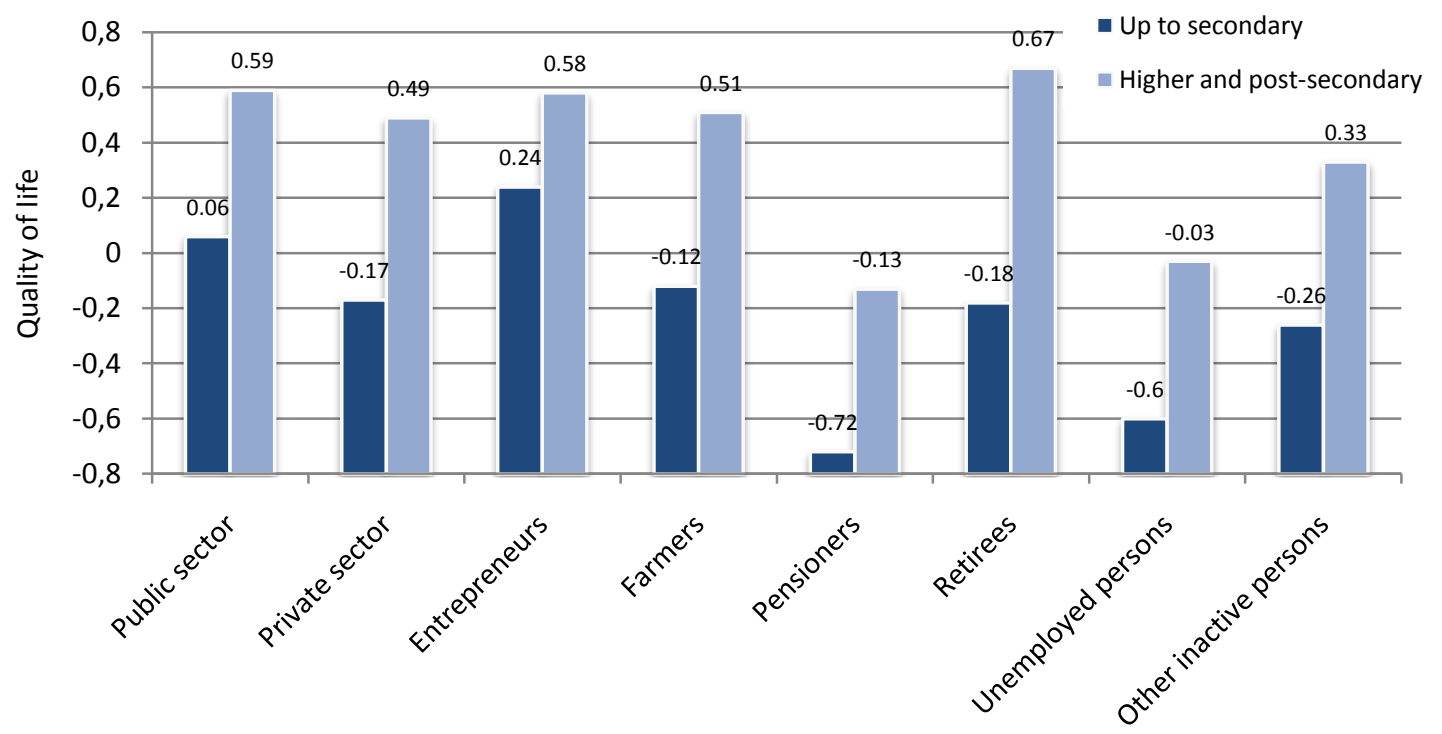

Social and professional status

NOTE: main effect of status $F(7,17324)=77.187, p<0.000, \eta^{2}=0.030$; main effect of education $F(1,17324)=445.988, p<0.000, \eta^{2}=$ 0.025 ; effect of interaction of status and education $F(7,17324)=9.322, p<0.000, \eta^{2}=0.004$.

Figure 9.2.3. General indicator of the quality of life depending on social and professional status and educational level, with age and gender control

Similarly to education, gender also modifies the differences in the quality of life of groups defined by social and professional status (Figure 9.2.4). In principle, there are no differences between men and women among private sector employees and school and university students. In the groups of public sector employees and retirees men enjoy a slightly better quality of life, but among pensioners, the unemployed and other professionally inactive women's quality of life is definitely better than that of men. Only in the group of public sector employees and pensioners do men prevail over women in terms of the quality of life. 


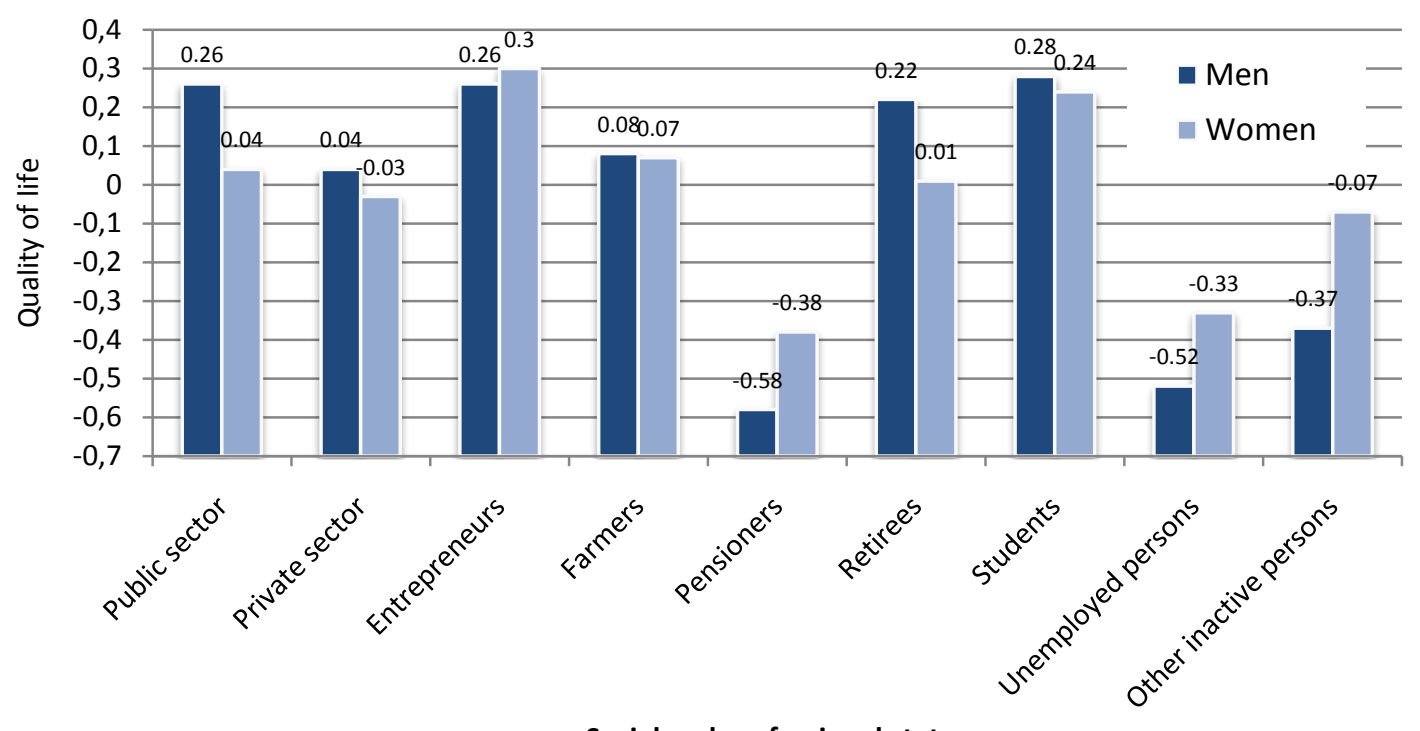

Social and professional status

NOTE: main effect of status $F(8,19328)=157.947, p<0.000, \eta^{2}=0.061$; main effect of gender $F<1$, ns; effect of interaction of status and gender $F(8,19722)=22.313, p<0.000, \eta^{2}=0.009$.

Figure 9.2.4. General indicator of the quality of life depending on social and professional status and gender, with age and educational level control

Gender and educational level, with age control, also play a significant role in explaining the difference in the quality of life of groups defined by marital status (Figures 9.2.5 and 9.2.6). Widowers feel definitely better and husbands slightly better than widows and wives respectively though divorce, albeit unfavourable for either gender, is more detrimental to men.

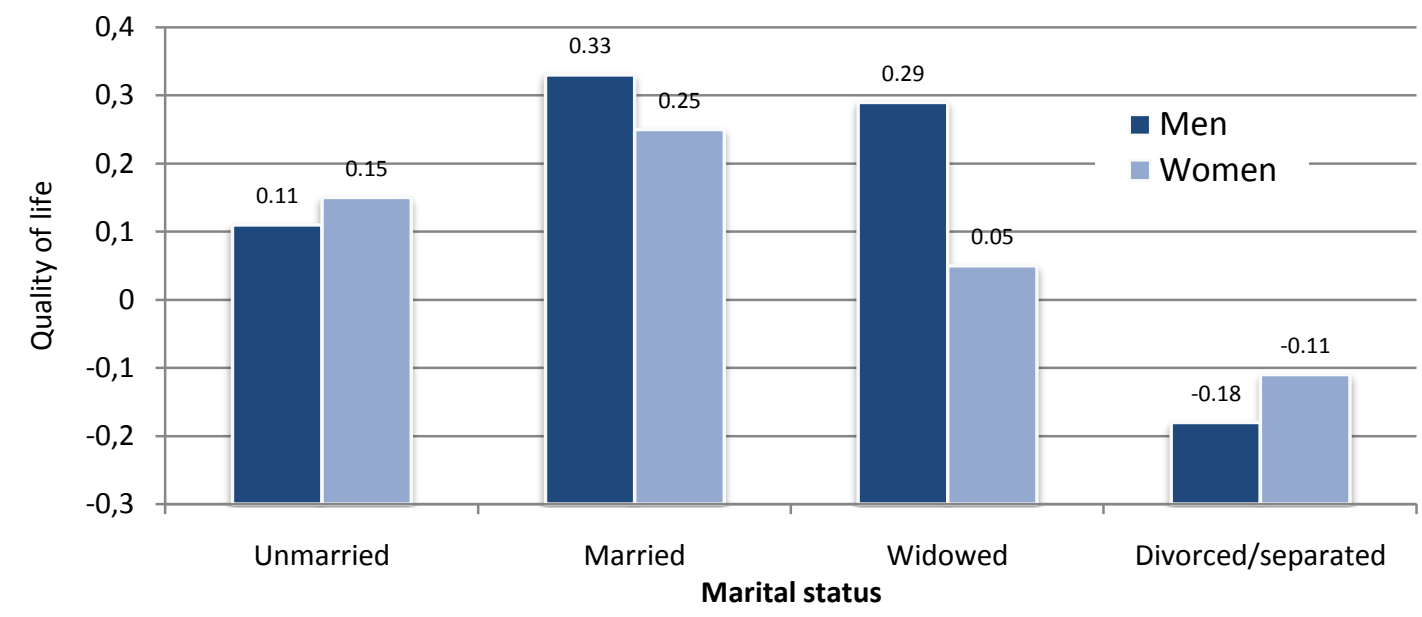

NOTE: main effect of marital status $F(3,19823)=62.071, p<0.000, \eta^{2}=0.009$; main effect of gender $F<4$, ns; the effect of interaction of marital status and gender $F(3,19823)=25.597, p<0.000, \eta^{2}=0.001$.

Figure 9.2.5. General indicator of the quality of life depending on marital status and gender, with age and educational level control

On the other hand, education nearly eliminates the differences in the quality of life that arise due to marital status (Figure 9.2.6). Although being widowed and divorced especially entails a significant decrease in the quality of life among persons with poorer education, a university diploma ensures that a high quality of life is retained also by those widowed and divorced. 


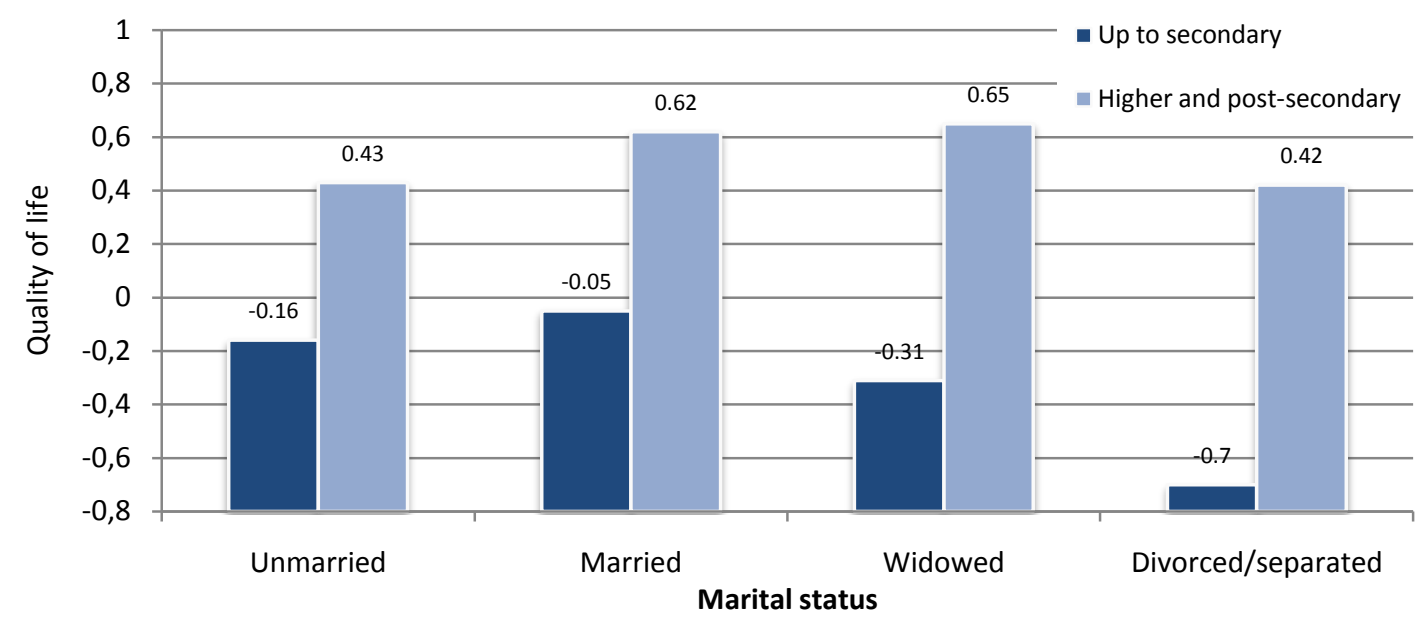

NOTE: main effect of marital status $F(3,19823)=62.071, p<0.000, \eta^{2}=0.009$; main effect of education $F(1,19823)=846.361, p<0.000$, $\eta^{2}=0.041$; the effect of interaction of marital status and education $F(3,19823)=20.207, p<0.000, \eta^{2}=0.003$.

Figure 9.2.6. General indicator of the quality of life depending on marital status and educational level, with age and gender control

Being male, and more importantly education, prevent a decrease in the quality of life after the partner's decease, primarily because these factors make it possible to maintain the material standard of living. This is corroborated by the effects of interaction of marital status with gender and educational level in terms of material well-being. After marriage breakdown, men fare better materially than when they were married, while the opposite is true for women: their material standard of living drops after divorce and even more so after the husband's decease (Figure 9.2.7). Also education protects material well-being after marriage breakdown (Figure 9.2.8). However, material well-being does not explain everything, as divorced men maintain their material standard of living to a greater extent than women but definitely lose on the general quality of life to a much greater extent than women, which is due to the worse level of other factors such as for example the level of pathology (mainly alcoholism).

The second-order effect of interaction of marital status, educational level and gender also proves to be significant (Figure 9.2.9). Higher education protects the material standard of living after marriage breakdown much more effectively among men than among women.

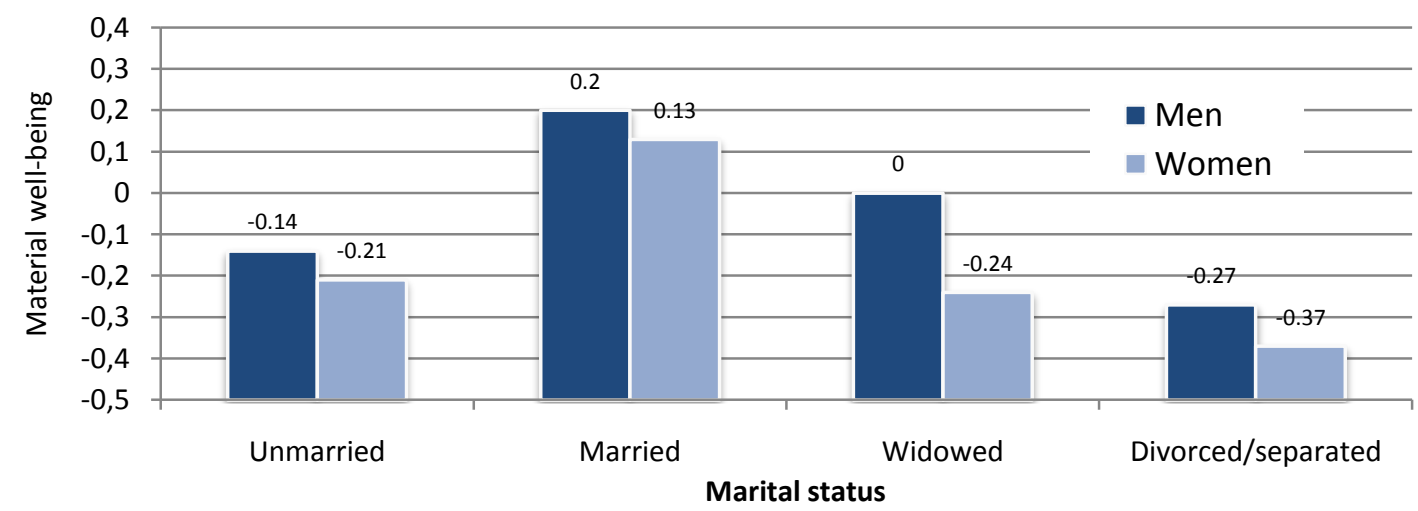

NOTE: main effect of marital status $F(3,24121)=265.220, p<0.000, \eta^{2}=0.032$; main effect of gender $F(1,24121)=38.766, p<0.000$, $\eta^{2}=0.002$; effect of interaction of marital status and gender $F(3,24121)=4.360, p<0.01, \eta^{2}=0.001$.

Figure 9.2.7. Material well-being depending on marital status and gender, with control for age and educational level 


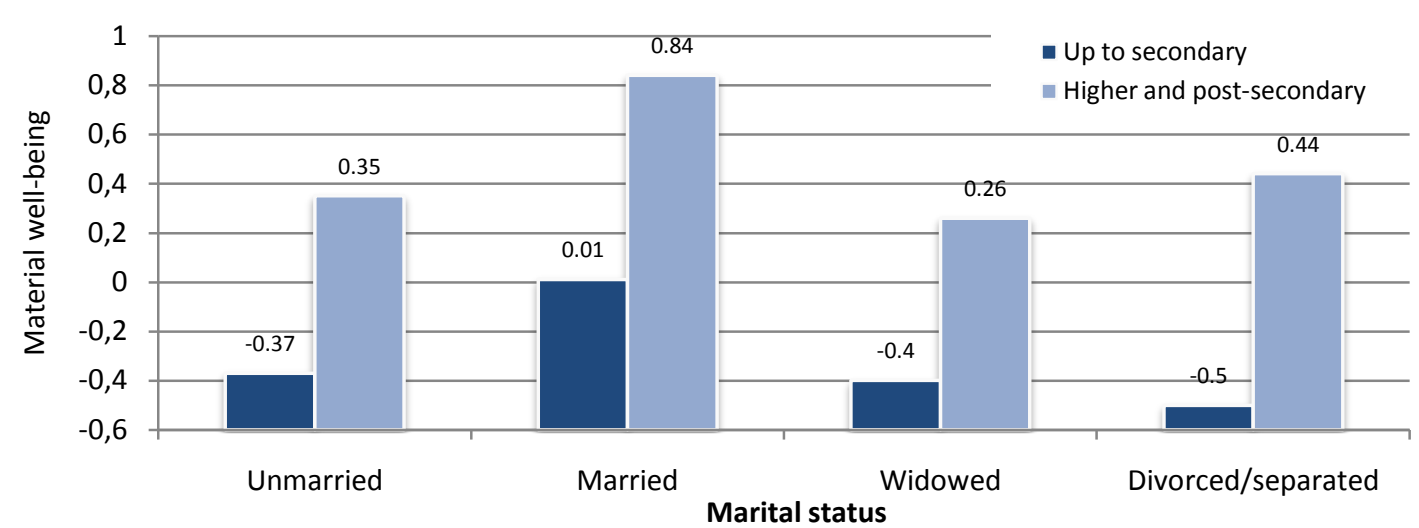

NOTE: main effect of marital status $F(3,24163)=248.220, p<0.000, \eta^{2}=0.030$; main effect of education $F(1,24163)=979.966$, $p<0.000, \eta^{2}=0.039$; effect of interaction of marital status and gender $F(3,24163)=8.512, p<0.000, \eta^{2}=0.001$.

Figure 9.2.8. Material well-being depending on marital status and educational level, with control for age and gender

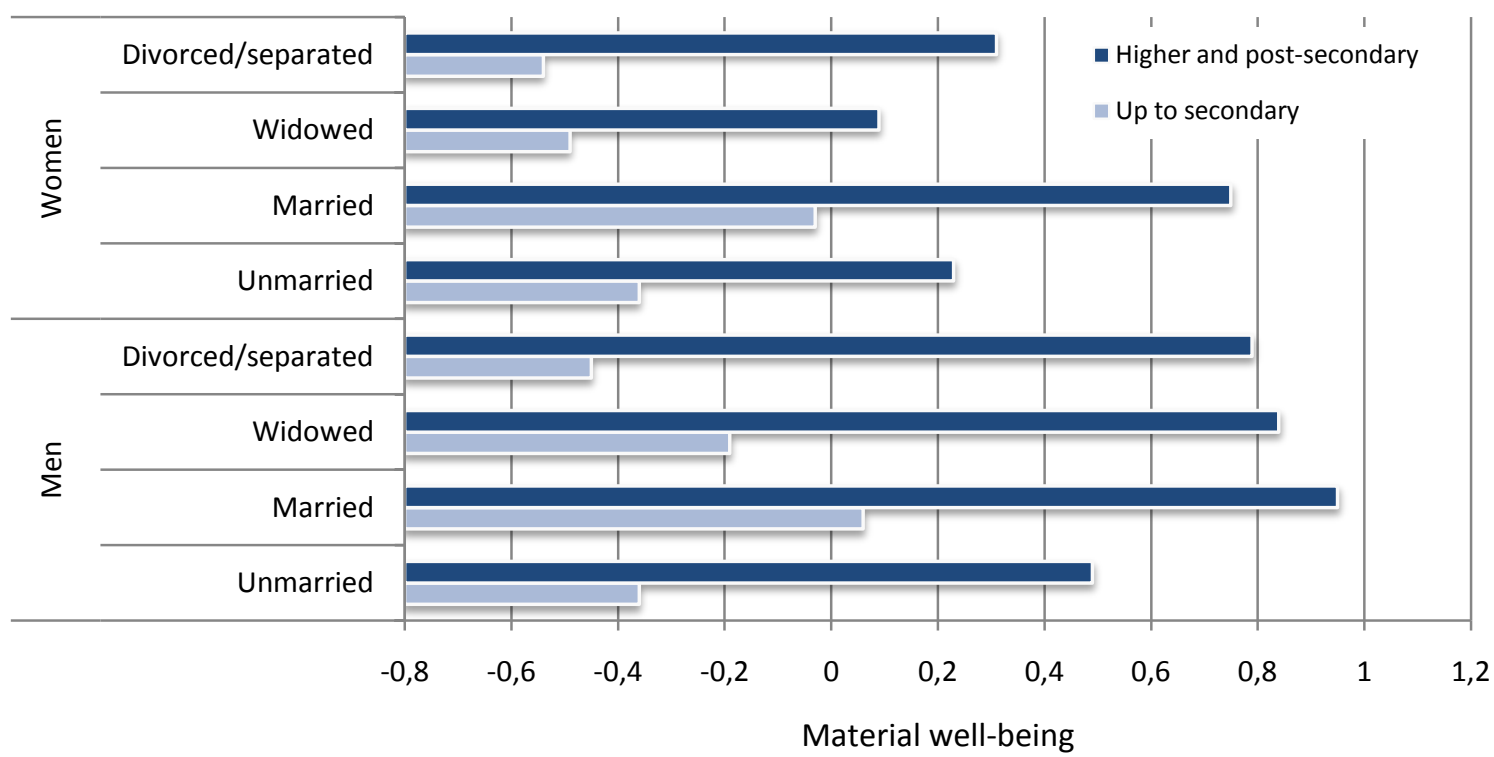

NOTE: main effect of marital status $F(3,24163)=248.220, p<0.000, \eta^{2}=0.030$; main effect of gender $F(1,24163)=96.066, p<0.000$, $\eta^{2}=0.004$; main effect of education $F(1,24163)=979.966, p<0.000, \eta^{2}=0.039$; effect of interaction of marital status, educational level and gender $F(3,24163)=3.834, p<0.01, \eta^{2}=0.000$.

Figure 9.2.9. Material well-being depending on marital status, educational level and gender, with control for age

When all previous factors and additionally the class of place of residence and bringing up children are taken into account in one multiple regression equation, we will be able to control the mutual relationships between those factors and thus better estimate the role of each of them as predictor (and perhaps even as determinant) of the quality of life and its individual dimensions. We carried out such analyses both for the general indicator of the quality of life and for eight component indicators. The results are presented in Tables 9.2.10 to 9.2.18.

The level of education is the best predictor of the general quality of life, which is independent of other factors $^{74}$, with age the second-best. Then there is unemployment, living on social security, marriage (a positive effect) and bringing up children (a negative effect). What also matters is divorce (negative effect), being an entrepreneur (positive effect), employment in the public sector (positive effect), employment in the private sector (negative effect), being widowed (negative effect), being a pensioner (negative effect) and gender (the quality of life is somewhat worse for women).

\footnotetext{
${ }^{74}$ It must be borne in mind however that the level of education was one of the variables taken into account in the civilisation level, a component of the quality of life.
} 
Table 9.2.10. Multiple regression analysis for general quality of life

\begin{tabular}{|c|c|c|c|c|c|}
\hline \multirow[t]{2}{*}{ Predictor } & \multicolumn{2}{|c|}{$\begin{array}{l}\text { Non-standardized } \\
\text { indicators }\end{array}$} & \multirow{2}{*}{$\begin{array}{c}\begin{array}{c}\text { Standardize } \\
\text { d indicator }\end{array} \\
\text { Beta }\end{array}$} & \multirow[t]{2}{*}{$t$} & \multirow[t]{2}{*}{ Significance } \\
\hline & B & Standard error & & & \\
\hline (Constant) & -0.507 & 0.047 & & 10.720 & 0.000 \\
\hline Education & 0.106 & 0.002 & 0.348 & 48.937 & 0.000 \\
\hline Age & -0.017 & 0.001 & -0.313 & 26.794 & 0.000 \\
\hline Gender (1 M, $2 \mathrm{~F})$ & -0.033 & 0.012 & -0.017 & -2.663 & 0.008 \\
\hline Class of place of residence (1 largest cities, 6 rural areas) & 0.001 & 0.004 & 0.001 & 0.191 & 0.848 \\
\hline Pensioners & -0.454 & 0.031 & -0.116 & $\begin{array}{r}- \\
14.666\end{array}$ & 0.000 \\
\hline Farmers & 0.009 & 0.035 & 0.002 & 0.270 & 0.787 \\
\hline Private sector employees & -0.041 & 0.019 & -0.018 & -2.126 & 0.034 \\
\hline Public sector employees & 0.109 & 0.024 & 0.037 & 4.565 & 0.000 \\
\hline Retirees & 0.087 & 0.029 & 0.035 & 3.058 & 0.002 \\
\hline Entrepreneurs & 0.238 & 0.033 & 0.049 & 7.279 & 0.000 \\
\hline Bringing up children & -0.236 & 0.017 & -0.116 & 14.213 & 0.000 \\
\hline Unemployed persons & -0.438 & 0.026 & -0.112 & 16.631 & 0.000 \\
\hline Marriage & 0.300 & 0.021 & 0.149 & 14.403 & 0.000 \\
\hline Widowed men/women & 0.130 & 0.030 & 0.040 & 4.270 & 0.000 \\
\hline Divorce & -0.258 & 0.032 & -0.056 & -8.004 & 0.000 \\
\hline
\end{tabular}

Bringing up children is the most significant predictor of stress in life (negative effect), followed by employment in the private sector (negative effect) and employment in the public sector (negative effect). More stress is also experienced by entrepreneurs, unemployed persons, married as well as divorced people, the elderly, those better educated and by men. Less stress is experienced by pensioners and widowed persons.

Table 9.2.11. Multiple regression analysis for stress in life

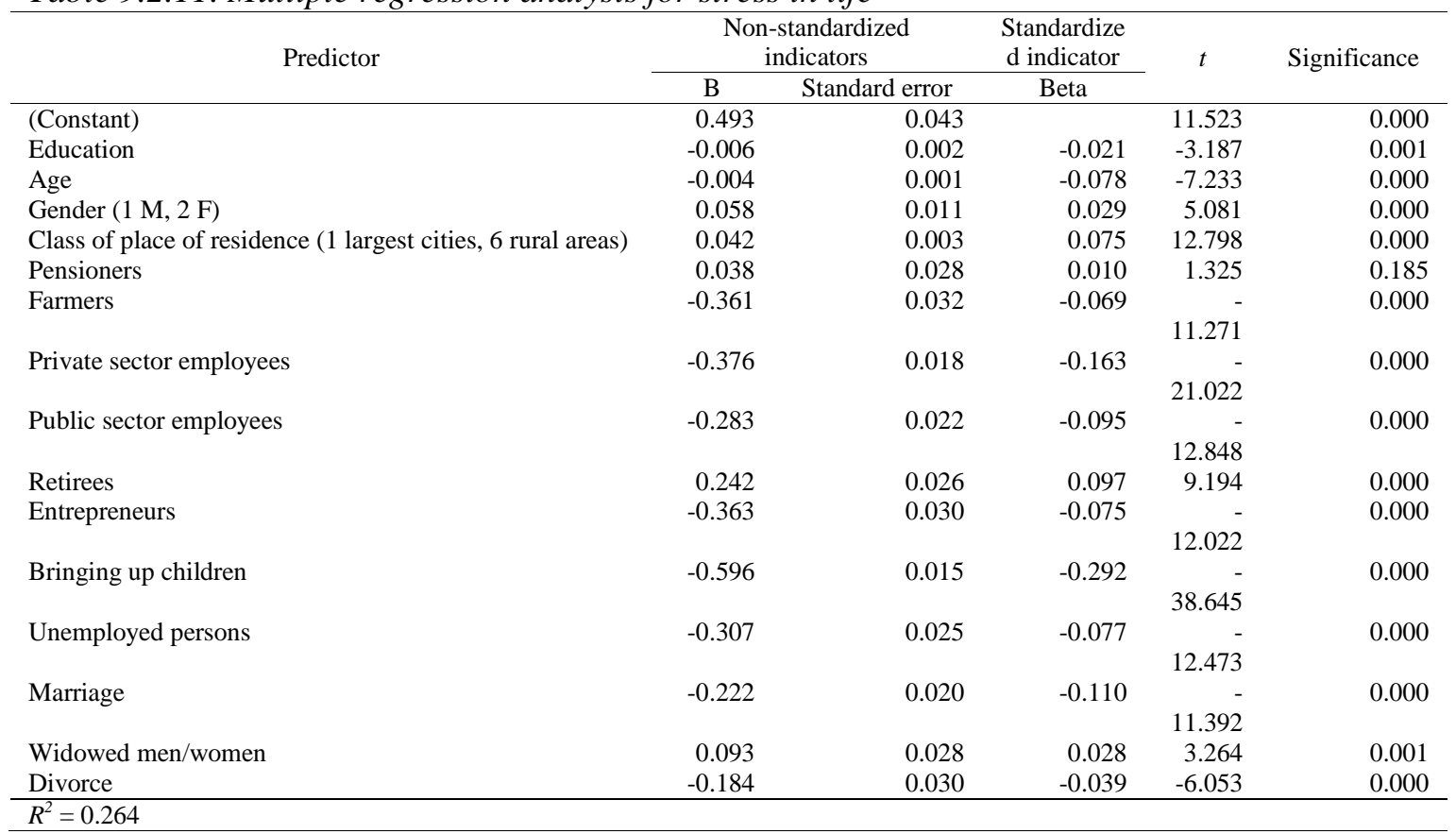

Age is the best predictor of psychological well-being (negative effect), followed by education (positive effect), marriage (positive effect), unemployment (negative effect) and divorce (negative effect). Also living on social security (negative effect), being an entrepreneur (positive effect), a pensioner or a public sector employee (positive effect) is significant. Bringing up children and being a woman is a moderately negative predictor of psychological well-being. These predictors explain nearly one-fourth of all variation in psychological well-being in the sample. 
Table 9.2.12. Multiple regression analysis for psychological well-being

\begin{tabular}{|c|c|c|c|c|c|}
\hline \multirow[t]{2}{*}{ Predictor } & \multicolumn{2}{|c|}{$\begin{array}{c}\text { Non-standardized } \\
\text { indicators }\end{array}$} & \multirow{2}{*}{$\begin{array}{c}\begin{array}{c}\text { Standardize } \\
\mathrm{d} \text { indicator }\end{array} \\
\text { Beta }\end{array}$} & \multirow[t]{2}{*}{$t$} & \multirow[t]{2}{*}{ Significance } \\
\hline & $\mathrm{B}$ & Standard error & & & \\
\hline (Constant) & 0.340 & 0.043 & & 7.916 & 0.000 \\
\hline Education & 0.047 & 0.002 & 0.155 & 23.486 & 0.000 \\
\hline \multirow[t]{2}{*}{ Age } & -0.021 & 0.001 & -0.391 & & 0.000 \\
\hline & & & & 35.507 & \\
\hline Gender (1 M, 2 F) & -0.033 & 0.012 & -0.016 & -2.799 & 0.005 \\
\hline Class of place of residence ( 1 largest cities, 6 rural areas) & -0.006 & 0.003 & -0.011 & -1.805 & 0.071 \\
\hline Pensioners & -0.243 & 0.029 & -0.062 & -8.363 & 0.000 \\
\hline Farmers & -0.001 & 0.032 & 0.000 & -0.017 & 0.987 \\
\hline Private sector employees & 0.027 & 0.018 & 0.012 & 1.481 & 0.139 \\
\hline Public sector employees & 0.077 & 0.022 & 0.026 & 3.440 & 0.001 \\
\hline Retirees & 0.127 & 0.027 & 0.051 & 4.733 & 0.000 \\
\hline Entrepreneurs & 0.207 & 0.031 & 0.043 & 6.736 & 0.000 \\
\hline Bringing up children & -0.089 & 0.016 & -0.044 & -5.681 & 0.000 \\
\hline \multirow[t]{2}{*}{ Unemployed persons } & -0.370 & 0.025 & -0.093 & & 0.000 \\
\hline & & & & 14.915 & \\
\hline Marriage & 0.323 & 0.020 & 0.160 & 16.273 & 0.000 \\
\hline Widowed men/women & 0.007 & 0.029 & 0.002 & 0.227 & 0.821 \\
\hline \multirow[t]{2}{*}{ Divorce } & -0.384 & 0.031 & -0.082 & - & 0.000 \\
\hline & & & & 12.424 & \\
\hline
\end{tabular}

Independently of all other factors, physical well-being is worse among the elderly, pensioners, women, retirees, divorcees and those with poorer education. Being a farmer, living in a rural area or small town, being an employee (irrespective of the sector), an entrepreneur and a widow(er) are positive predictors. Also, employees (especially in the private sector) and entrepreneurs are healthier than the general population. More than one-third of variation in physical well-being is related to that set of predictors.

Table 9.2.13. Multiple regression analysis for physical well-being

\begin{tabular}{|c|c|c|c|c|c|}
\hline \multirow[t]{2}{*}{ Predictor } & \multicolumn{2}{|c|}{$\begin{array}{c}\text { Non-standardized } \\
\text { indicators }\end{array}$} & \multirow{2}{*}{$\begin{array}{c}\begin{array}{c}\text { Standardize } \\
\text { d indicator }\end{array} \\
\text { Beta }\end{array}$} & \multirow[t]{2}{*}{$t$} & \multirow[t]{2}{*}{ Significance } \\
\hline & $\mathrm{B}$ & Standard error & & & \\
\hline (Constant) & 0.683 & 0.040 & & 17.073 & 0.000 \\
\hline Education & 0.022 & 0.002 & 0.073 & 11.817 & 0.000 \\
\hline Age & -0.019 & 0.001 & -0.339 & 32.861 & 0.000 \\
\hline Gender (1 M, 2 F) & -0.085 & 0.011 & -0.042 & -7.800 & 0.000 \\
\hline Class of place of residence ( 1 largest cities, 6 rural areas) & 0.017 & 0.003 & 0.030 & 5.283 & 0.000 \\
\hline Pensioners & -1.136 & 0.027 & -0.289 & 41.943 & 0.000 \\
\hline Farmers & 0.143 & 0.030 & 0.028 & 4.741 & 0.000 \\
\hline Private sector employees & 0.141 & 0.017 & 0.061 & 8.312 & 0.000 \\
\hline Public sector employees & 0.115 & 0.021 & 0.038 & 5.489 & 0.000 \\
\hline Retirees & -0.206 & 0.025 & -0.082 & -8.203 & 0.000 \\
\hline Entrepreneurs & 0.215 & 0.029 & 0.044 & 7.472 & 0.000 \\
\hline Bringing up children & 0.000 & 0.015 & 0.000 & -0.007 & 0.994 \\
\hline Unemployed persons & 0.011 & 0.023 & 0.003 & 0.480 & 0.631 \\
\hline Marriage & -0.010 & 0.019 & -0.005 & -0.539 & 0.590 \\
\hline Widowed men/women & 0.089 & 0.027 & 0.027 & 3.280 & 0.001 \\
\hline Divorce & -0.097 & 0.029 & -0.021 & -3.363 & 0.001 \\
\hline
\end{tabular}

The level of social capital is determined first and foremost by the level of education. The paradox however is that despite the fast growth in the number of people with higher education, social capital is not growing just as fast (see section 6.3). Slightly less significant but still fairly important are such factors as age (positive effect), gender (men score higher), class of place of residence (the smaller the town, the higher the level of social capital), being a farmer (positive effect), employment in the private sector (negative effect), employment in the public sector (positive effect), being an entrepreneur (weak positive effect), bringing up children (positive effect) and divorce (weak negative effect). Summed up, all these predictors explain only $11 \%$ of variation in the value of the standardized indicator of social capital. 
Table 9.2.14. Multiple regression analysis for social capital

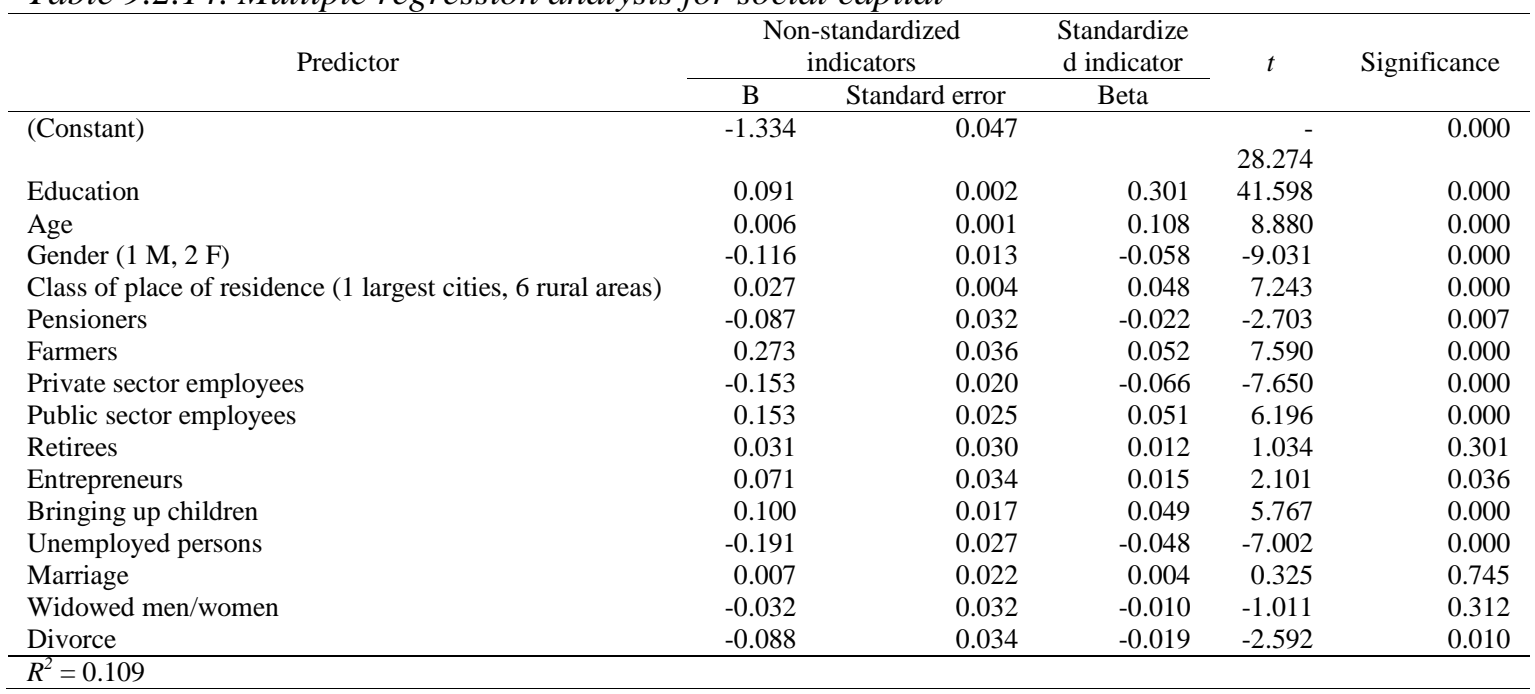

The incidence of pathology diminishes with age and education, but gender is its strongest predictor: the pathology indicator is much higher among men than among women. The larger the place of residence, the more pathologies there are. Unemployment, divorce and bringing up children increase pathology while marriage, employment in the public sector and being a farmer diminish it. Only $5 \%$ of variation in that indicator of the quality of life is explained by all the predictors.

Table 9.2.15. Multiple regression analysis for pathology (reversed scale)

\begin{tabular}{|c|c|c|c|c|c|}
\hline \multirow[t]{2}{*}{ Predictor } & \multicolumn{2}{|c|}{$\begin{array}{c}\text { Non-standardized } \\
\text { indicators }\end{array}$} & \multirow{2}{*}{$\begin{array}{c}\begin{array}{c}\text { Standardize } \\
\text { d indicator }\end{array} \\
\text { Beta }\end{array}$} & \multirow[t]{2}{*}{$t$} & \multirow[t]{2}{*}{ Significance } \\
\hline & B & Standard error & & & \\
\hline (Constant) & -0.989 & 0.047 & & 20.895 & 0.000 \\
\hline Education & 0.010 & 0.002 & 0.034 & 4.664 & 0.000 \\
\hline Age & 0.004 & 0.001 & 0.065 & 5.313 & 0.000 \\
\hline Gender (1 M, 2 F) & 0.299 & 0.013 & 0.149 & 23.254 & 0.000 \\
\hline Class of place of residence (1 largest cities, 6 rural areas) & 0.043 & 0.004 & 0.077 & 11.598 & 0.000 \\
\hline Pensionersy & -0.006 & 0.032 & -0.002 & -.199 & 0.842 \\
\hline Farmers & 0.113 & 0.036 & 0.022 & 3.165 & 0.002 \\
\hline Private sector employees & 0.025 & 0.020 & 0.011 & 1.236 & 0.216 \\
\hline Public sector employees & 0.104 & 0.025 & 0.035 & 4.229 & 0.000 \\
\hline Retirees & 0.103 & 0.030 & 0.041 & 3.484 & 0.000 \\
\hline Entrepreneurs & -0.044 & 0.034 & -0.009 & -1.302 & 0.193 \\
\hline Bringing up children & -0.070 & 0.017 & -0.034 & -4.039 & 0.000 \\
\hline Unemployed persons & -0.157 & 0.027 & -0.040 & -5.736 & 0.000 \\
\hline Marriage & 0.120 & 0.022 & 0.060 & 5.487 & 0.000 \\
\hline Widowed men/women & 0.035 & 0.032 & 0.011 & 1.086 & 0.278 \\
\hline Divorce & -0.200 & 0.034 & -0.042 & -5.803 & 0.000 \\
\hline
\end{tabular}

The greatest proportion of variation in material well-being is explained by educational level. Education remains the most reliable guarantor of affluence (cf. section 5.5.3). People in rural areas continue to be worse-off that residents of towns, while inhabitants of small towns are worse-off than those who live in the large towns. Also marriage, as a community which accumulates material goods, is a strong predictor. It is not surprising that entrepreneurs are significantly better-off than others though also employees, both in the public and in the private sector, turn out to be better-off especially when compared to unemployed persons. Bringing up children is costly and thus diminishes the family's material well-being. Also divorce negatively affects well-being and so does being pensioner. On the other hand, widowers and widows are better-off; also men fare better, as already discussed in the chapter on discrimination (8.3). Even though living in a rural area entails a lower average material standard, this generally does not concern farmers; these do not depart from the national average in terms of material well-being. This does not mean that they do not obtain lower incomes, yet they may own more goods, which to some extent compensates for lower income. The predictors included in the regression equation explain a total of nearly $29 \%$ of variation in material wellbeing. 
Table 9.2.16. Multiple regression analysis for material well-being

\begin{tabular}{|c|c|c|c|c|c|}
\hline \multirow[t]{2}{*}{ Predictor } & \multicolumn{2}{|c|}{$\begin{array}{l}\text { Non-standardized } \\
\text { indicators }\end{array}$} & \multirow{2}{*}{$\begin{array}{c}\begin{array}{c}\text { Standardize } \\
\text { d indicator }\end{array} \\
\text { Beta }\end{array}$} & \multirow[t]{2}{*}{$t$} & \multirow[t]{2}{*}{ Significance } \\
\hline & B & Standard error & & & \\
\hline (Constant) & -0.584 & 0.042 & & - & 0.000 \\
\hline & & & & 13.906 & \\
\hline Age & -0.009 & 0.001 & $\begin{array}{r}0.014 \\
-0.156\end{array}$ & - & 0.000 \\
\hline & & & & 14.382 & \\
\hline Gender (1 M, 2 F) & -0.038 & 0.011 & -0.019 & -3.309 & 0.001 \\
\hline Class of place of residence ( 1 largest cities, 6 rural areas) & -0.082 & 0.003 & -0.146 & 24.982 & 0.000 \\
\hline Pensionersy & -0.148 & 0.028 & -0.038 & -5.253 & 0.000 \\
\hline Farmers & -0.008 & 0.032 & -0.002 & -0.255 & 0.799 \\
\hline Private sector employees & 0.132 & 0.018 & 0.057 & 7.397 & 0.000 \\
\hline Public sector employees & 0.190 & 0.022 & 0.063 & 8.593 & 0.000 \\
\hline Retirees & 0.009 & 0.026 & 0.004 & 0.332 & 0.740 \\
\hline Entrepreneurs & 0.575 & 0.031 & 0.116 & 18.801 & 0.000 \\
\hline Bringing up children & -0.216 & 0.015 & -0.106 & - & 0.000 \\
\hline Unemployed persons & -0.392 & 0.024 & -0.099 & $\begin{array}{r}14.084 \\
- \\
16.086\end{array}$ & 0.000 \\
\hline Marriage & 0.465 & 0.019 & 0.231 & 23.905 & 0.000 \\
\hline Widowed men/women & 0.118 & 0.028 & 0.036 & 4.187 & 0.000 \\
\hline Divorce & -0.091 & 0.030 & -0.019 & -3.002 & 0.003 \\
\hline
\end{tabular}

Marriage and young age ensure the greatest social support. Divorce negatively affects social well-being and so does bringing up children and unemployment. Pensioners, men and entrepreneurs enjoy greater social support than others. Also educational level is favourable for social well-being although to a limited extent. In general however only $4 \%$ of variation in the value of that indicator of the quality of life is explained, which is the lowest of all proportions.

Table 9.2.17. Multiple regression analysis for social well-being

\begin{tabular}{|c|c|c|c|c|c|}
\hline \multirow[t]{2}{*}{ Predictor } & \multicolumn{2}{|c|}{$\begin{array}{l}\text { Non-standardized } \\
\text { indicators }\end{array}$} & \multirow{2}{*}{$\begin{array}{c}\begin{array}{c}\text { Standardize } \\
\mathrm{d} \text { indicator }\end{array} \\
\text { Beta }\end{array}$} & \multirow[t]{2}{*}{$t$} & \multirow[t]{2}{*}{ Significance } \\
\hline & $\mathrm{B}$ & Standard error & & & \\
\hline (Constant) & 0.197 & 0.048 & & 4.117 & 0.000 \\
\hline Education & 0.010 & 0.002 & 0.032 & 4.376 & 0.000 \\
\hline Age & -0.009 & 0.001 & -0.166 & - & 0.000 \\
\hline & & & & 13.403 & \\
\hline Gender (1 M, 2 F) & -0.046 & 0.013 & -0.023 & -3.527 & 0.000 \\
\hline Class of place of residence ( 1 largest cities, 6 rural areas) & 0.006 & 0.004 & 0.010 & 1.509 & 0.131 \\
\hline Pensioners & -0.020 & 0.032 & -0.005 & -.630 & 0.529 \\
\hline Farmers & 0.052 & 0.036 & 0.010 & 1.422 & 0.155 \\
\hline Private sector employees & -0.009 & 0.020 & -0.004 & -.468 & 0.640 \\
\hline Public sector employees & 0.043 & 0.025 & 0.014 & 1.708 & 0.088 \\
\hline Retirees & 0.211 & 0.030 & 0.085 & 7.061 & 0.000 \\
\hline Entrepreneurs & 0.113 & 0.034 & 0.023 & 3.273 & 0.001 \\
\hline Bringing up children & -0.129 & 0.018 & -0.063 & -7.354 & 0.000 \\
\hline Unemployed persons & -0.196 & 0.028 & -0.049 & -7.059 & 0.000 \\
\hline Marriage & 0.303 & 0.022 & 0.150 & 13.630 & 0.000 \\
\hline Widowed men/women & -0.037 & 0.032 & -0.011 & -1.144 & 0.253 \\
\hline Divorce & -0.284 & 0.035 & -0.060 & -8.194 & 0.000 \\
\hline
\end{tabular}

In the regression analysis of civilisation level, education was removed from the list of predictors as it was already one of the components of that indicator. This gives the role of the strongest predictor to age, a strong negative correlate of the level of education. Employment, especially in the public sector, is also very important. Positive influence is exerted by being an entrepreneur or pensioner (obviously, after excluding the age effect), being married and bringing up children; a negative effect is characteristic of unemployment, living on social security, being a widow(er) and a farmer. Civilisation level is very strongly differentiated by the size of place of residence: the smaller it is, the lower the civilisation level. Overall, the predictors included in the regression equation explain nearly half of the variance of the civilisation level indicator. 
Table 9.2.18. Multiple regression analysis for the civilisation level

\begin{tabular}{|c|c|c|c|c|c|}
\hline \multirow[t]{2}{*}{ Predictor } & \multicolumn{2}{|c|}{$\begin{array}{l}\text { Non-standardized } \\
\text { indicators }\end{array}$} & \multirow{2}{*}{$\begin{array}{c}\begin{array}{c}\text { Standardize } \\
\text { d indicator }\end{array} \\
\text { Beta }\end{array}$} & \multirow[t]{2}{*}{$t$} & \multirow[t]{2}{*}{ Significance } \\
\hline & B & Standard error & & & \\
\hline (Constant) & 1.990 & 0.026 & & 77.810 & 0.000 \\
\hline Age & -0.032 & 0.000 & -0.571 & -65.303 & 0.000 \\
\hline Gender (1 M, 2 F) & -0.068 & 0.009 & -0.034 & -7.182 & 0.000 \\
\hline Class of place of residence ( 1 largest cities, 6 rural areas) & -0.134 & 0.003 & -0.239 & -51.218 & 0.000 \\
\hline Pensioners & -0.149 & 0.023 & -0.038 & -6.343 & 0.000 \\
\hline Farmers & -0.069 & 0.026 & -0.014 & -2.636 & 0.008 \\
\hline Private sector employees & 0.216 & 0.015 & 0.094 & 14.724 & 0.000 \\
\hline Public sector employees & 0.561 & 0.017 & 0.189 & 32.096 & 0.000 \\
\hline Retirees & 0.100 & 0.022 & 0.040 & 4.596 & 0.000 \\
\hline Entrepreneurs & 0.556 & 0.025 & 0.116 & 22.505 & 0.000 \\
\hline Bringing up children & 0.062 & 0.013 & 0.030 & 4.852 & 0.000 \\
\hline Unemployed persons & -0.140 & 0.020 & -0.035 & -6.881 & 0.000 \\
\hline Marriage & 0.189 & 0.016 & 0.094 & 11.777 & 0.000 \\
\hline Widowed men/women & -0.078 & 0.023 & -0.024 & -3.331 & 0.001 \\
\hline Divorce & 0.024 & 0.025 & 0.005 & 0.966 & 0.334 \\
\hline$R^{2}=0.492$ & & & & & \\
\hline
\end{tabular}

The criteria of the quality of life adopted here are not fully objective but also a good life is also simply a happy life not just what meets some objective standards (of affluence, health, respect, etc.) and it remains an open question what could measure the truth in respect this respect. Researchers from diverse fields of science have debated on that topic for years (cf. Czapiński, 2002b, 2004b; Lewicka, 2005), which in the last decade gave rise to the development of a new branch of study called positive psychology. 


\subsection{Is Polish society becoming more and more stratified?}

In the opinion of many economists, economic growth of a relatively poor country should entail its greater socio-economic stratification. Indeed, throughout the period when Social Diagnosis has been carried out, the proportion of income of the richest $20 \%$ of households to that of the poorest $20 \%$ increased until 2009. It is worth noting that the growing stratification resulted first and foremost from the higher growth rate of the highest incomes (Figure 9.3.1). The value of the 9th decile of household income per equivalent unit in terms of constant prices of 2000 increased by $45.6 \%$ between 2000 and 2009, which is much more than the average for entire samples $(38.9 \%$ ), while the value of the 1 st decile increased by $27.6 \%$ over that period. However, over the last two years the increase in 1st decile real income was greater than the growth of the 9th decile for the first time since the beginning of the survey ( $8 \%$ and $4 \%$ respectively) and thus the proportion of the 9 th to the 1 st decile diminished (Table 9.3.1).

Thus, Poles have not been getting richer at the same pace. This, however, does not mean that the poor have had fewer opportunities for economic advancement than the rich. Quite on the contrary, while the income scale increased, poor households were catching up on the richer ones. The income of the poorest $10 \%$ of households grew at a much faster pace over the past four years and slightly faster over the last two years than the income of the richest $10 \%$ of households (Figure 9.3.2) ${ }^{75}$.

Table 9.3.1. Variation in household net income in entire samples between 2000 and 2011

\begin{tabular}{|c|c|c|c|c|}
\hline \multirow[t]{2}{*}{ Study year } & \multicolumn{2}{|c|}{$\begin{array}{l}\text { Ratio of the 4th to the 1st quintile of household } \\
\text { income }\end{array}$} & \multicolumn{2}{|c|}{$\begin{array}{c}\text { Ratio of the 9th to the 1st decile of household } \\
\text { income }\end{array}$} \\
\hline & Total & Per equivalent unit & Total & Per equivalent unit \\
\hline 2000 & 3.64 & 2.15 & 4.48 & 3.59 \\
\hline 2003 & 2.82 & 2.33 & 4.62 & 3.94 \\
\hline 2005 & 2.80 & 2.36 & 4.67 & 3.98 \\
\hline 2007 & 3.00 & 2.41 & 5.12 & 4.15 \\
\hline 2009 & 3.15 & 2.48 & 5.56 & 4.10 \\
\hline 2011 & 3.00 & 2.46 & 5.28 & 3.96 \\
\hline \multicolumn{5}{|l|}{ Difference between: } \\
\hline 2011 and 2000 & 0.36 & 0.31 & 0.80 & 0.37 \\
\hline 2011 and 2009 & -0.15 & -0.02 & -0.28 & -0.14 \\
\hline
\end{tabular}

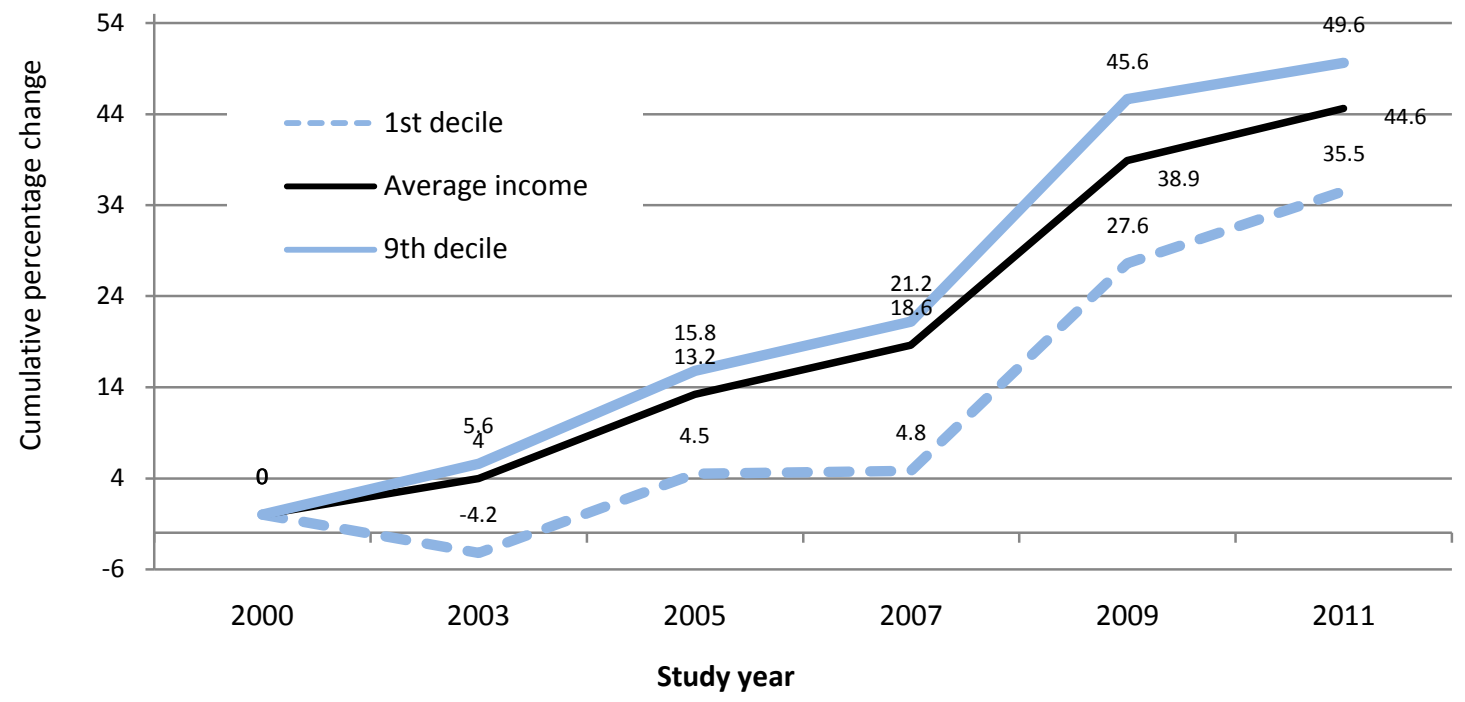

Figure 9.3.1. Cumulative percentage change in the middle, 1st and 9th decile of household income per equivalent unit in the previous month in terms of constant prices of the year 2000 between 2000 and 2011

\footnotetext{
${ }^{75}$ The objection that this is an instance of the base effect (an increase in nominal income by X yields a greater percentage growth when the initial level is low than when it is high) may be countered by stating that irrespective of the base effect this means that income differences between the rich and the poor have been diminishing rather than growing. Moreover, the base effect does not explain why the smallest percentage increases in income were found in the groups of households with middle income (the $3 \mathrm{rd}$ and 7 th decile).
} 


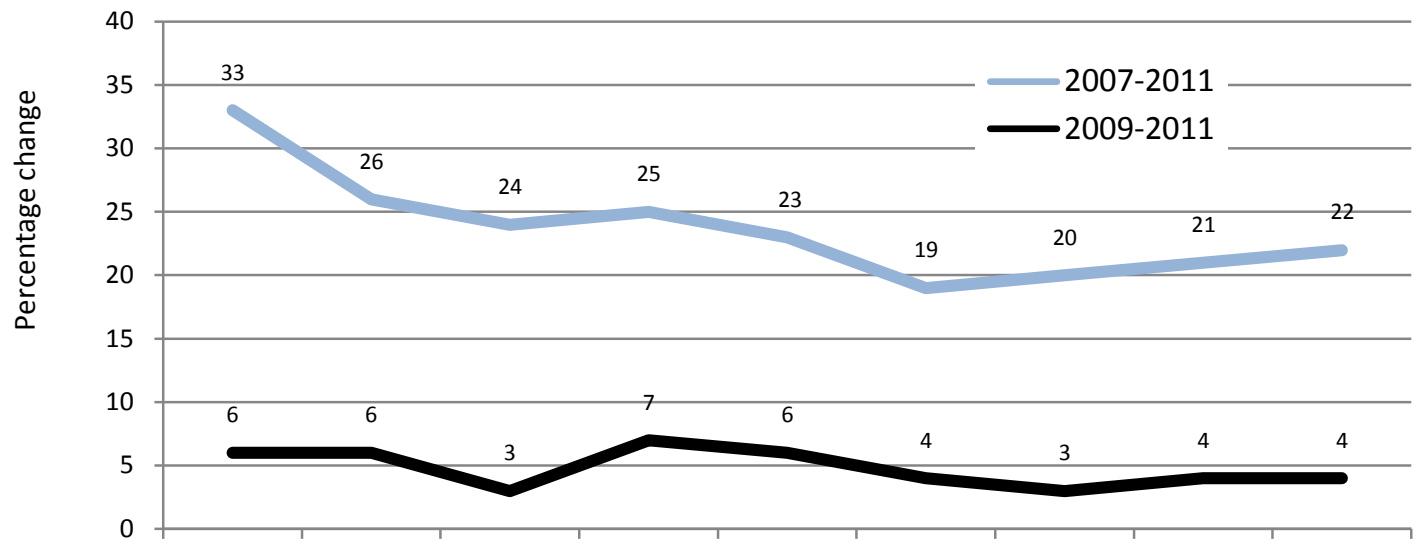

1st decile 2 nd decile 3 rd decile 4th decile 5th decile 6th decile 7th decile 8 th decile 9th decile

\section{Household groups by income}

Figure 9.3.2. Percentage change in household income per equivalent unit in terms of constant prices of 2000 in the years 2007-2011 and 2009-2011 in groups of households by income deciles in panel samples

The statement that Polish society is increasingly stratified in economic terms is proved false by the symmetric, two-directional mobility of households on the income axis. Only 59\% of households from the group of the $20 \%$ who were the poorest in 2007 remained in that group after four years and exactly the same proportion from the group of the richest $20 \%$ remained in that group in 2011 . Thus, $41 \%$ of the poorest moved to higher income groups (a majority of $23 \%$ only moved one level up) and $41 \%$ of the richest moved to lower income groups (a majority of $23 \%$ moved one quintile down). With the shorter time span of two years between 2009 and 2011, 80\% remained in the lowest group of households and $90 \%$ remained in the top group. Thus we have a nearly full (and after four years a perfectly full) symmetry of the changes in the position of the richest $20 \%$ and the poorest $20 \%$ of households in terms of income.

The economic distance between the poorest and the richest households that remained in their income groups basically did not change over four and two years (Figures 9.3.3 and 9.3.4), while in the case of households that did change position in terms of income, the distance diminished 12 times over four years and 4 times over the past two years. 


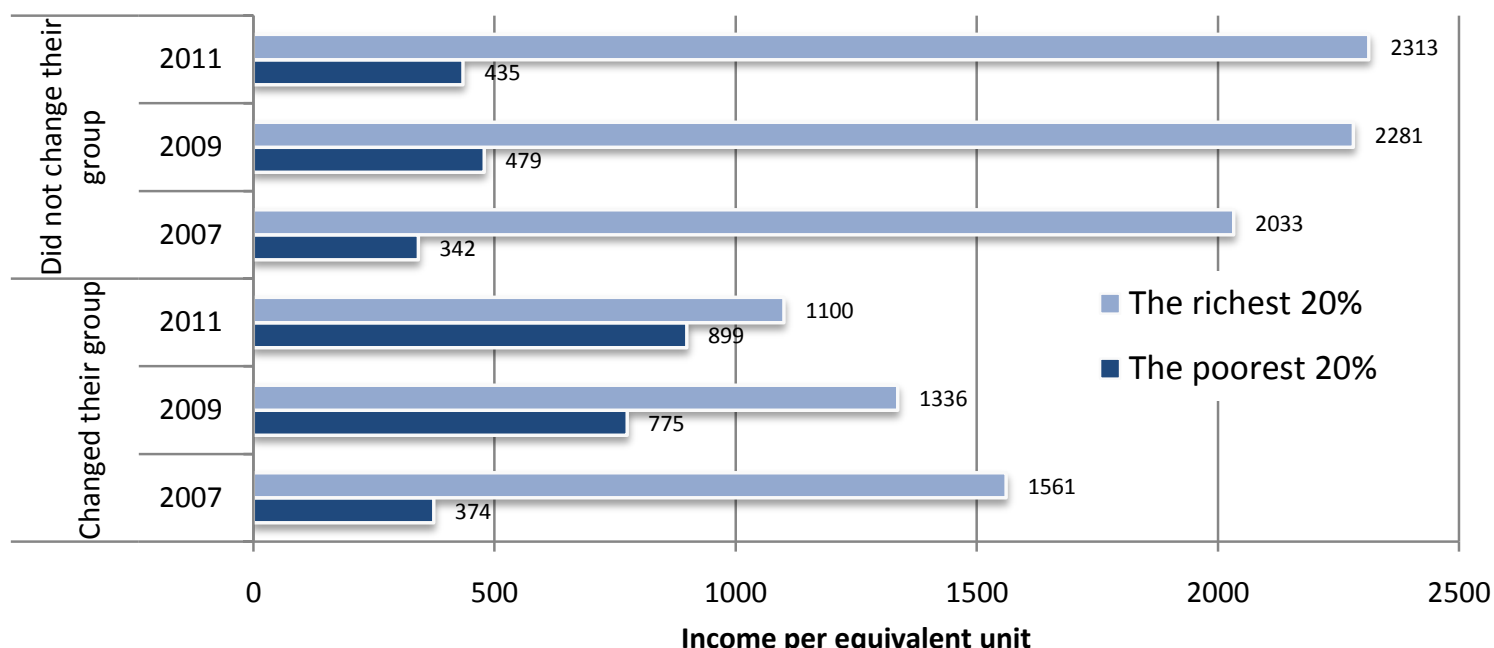

NOTE: effect of changing the group $F(1,871)=49.463, p<0.000, \eta^{2}=0.054$; effect of the date of measurement $F(2,870)=21.063, p<0.000$, $\eta^{2}=0.046$; effect of interaction of changing the group, initial group and date of measurement $F(2,870)=80.078 ; p<0.000, \eta^{2}=0.155$; effect of interaction of the initial group and the date of measurement $F(2,870)=37.363, p<0.000, \eta^{2}=0.079$; effect of interaction of changing the group and the date of measurement $F(2,870)=5.703, p<0.000, \eta^{2}=0.013$.

Figure 9.3.3. Household income per equivalent unit in 2007, 2009 and 2011 in terms of constant prices of the year 2000 in the group of the poorest $20 \%$ and the richest $20 \%$ of households by equivalent per capita income quintiles in 2007 which in 2011 remained or did not remain in the same quintile groups in the panel sample

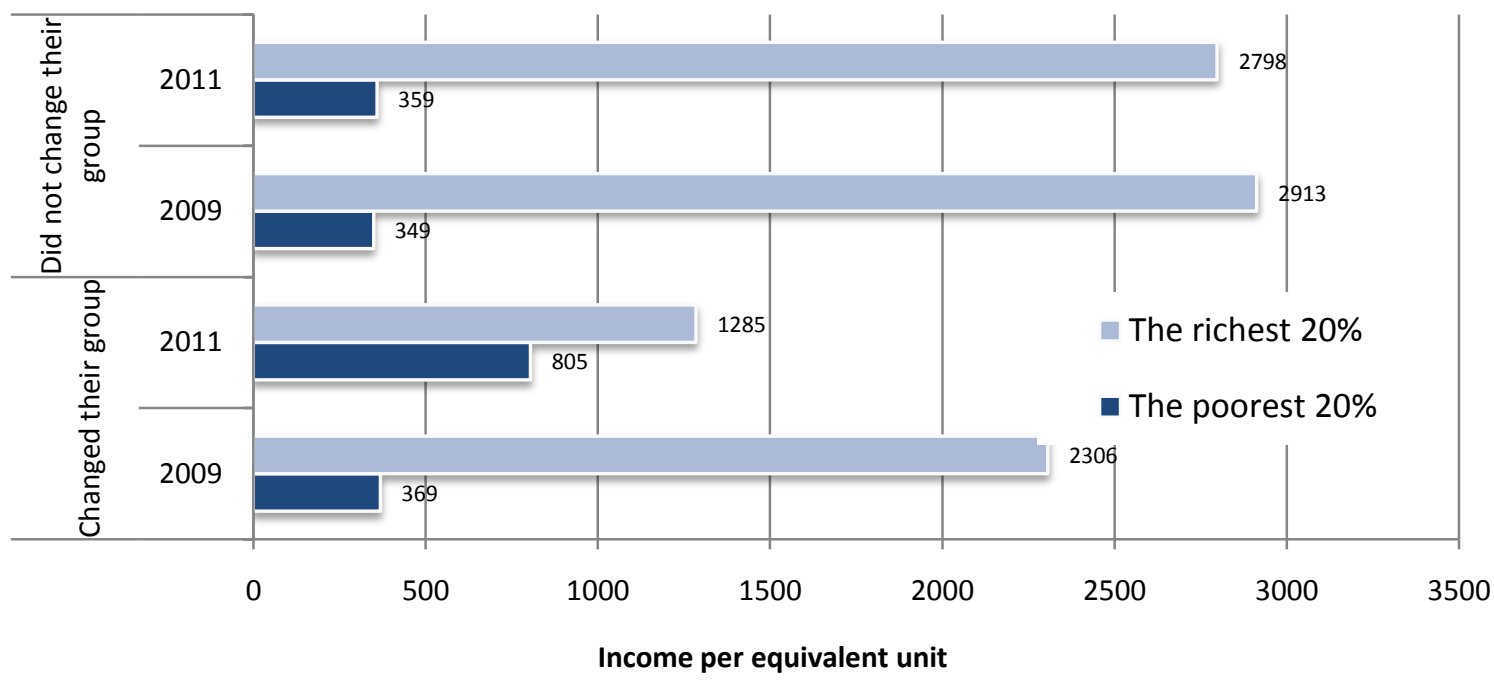

NOTE: effect of changing the group $F(1,1681)=154.363, p<0.000, \eta^{2}=0.084$; effect of the date of measurement $F(1,1681)=79.405$, $p<0.000, \eta^{2}=0.045$; effect of interaction of changing the group, the initial group and the date of measurement $F(1,1681)=296.441$; $p<0.000, \eta^{2}=0.150$; effect of interaction of the initial group and date of measurement $F(1,1681)=418.063, p<0.000, \eta^{2}=0.199$; effect of interaction of changing the group and of the date of measurement $F(1,1681)=38.547, p<0.000, \eta^{2}=0.022$.

Figure 9.3.4. Household income per equivalent unit in 2009 and 2011 in terms of constant prices of the year2000 in the group of the poorest $20 \%$ and the richest $20 \%$ of households by equivalent per capita income quintiles in 2009 which in 2011 remained or did not remain in the same quintile groups in the panel sample

One may add that the difference between the groups on the extremes in terms of the standardized indicator of the quality of life was nearly the same in 2009 as two years before (2.1 and 2.07 of standard deviation) and between 2005 and 2009 the difference diminished by 0.3 of standard deviation (Table 9.1.2). This suggests that the distance between the social groups with the highest and the lowest quality of life is not increasing. The Poles are improving their quality of life together rather than at each other's expense. 